\title{
On the Formation and On the Surface of Inorganic Lead, Barium and Antimony Based Gunshot Residues: A Thermodynamic Approach
}

\author{
Felice Nunziata ${ }^{* 1}$ and Marco Morin ${ }^{2}$ \\ ${ }^{1}$ ARPAC (Campania’s Regional Environmental Protection Agency) Naples Department, Member of The Chartered \\ Forensic Science Society, Italy \\ ${ }^{2}$ Member of The Chartered Forensic Science Society, Founder member of the International Association of Maritime \\ Studies, Italy
}

${ }^{*}$ Corresponding author: Felice Nunziata, ARPAC (Campania's Regional Environmental Protection Agency) Naples Department, Member of The Chartered Forensic Science Society, Italy, E-mail: trebiconlab@inwind.it

Citation: Felice Nunziata, Marco Morin (2017) On The Formation and On the Surface of Inorganic Lead, Barium and Antimony Based Gunshot Residues: A Thermodynamic Approach. J Forensic Sci Criminol 5(3): 303

Received Date: May 06, 2016 Accepted Date: June 30, 2017 Published Date: July 03, 2017

\begin{abstract}
In their first full report on the analysis of GSR by SEM-EDS, Wolten and colleagues stated that the great majority of the gunshot residue particles (GSR), between 70 to $100 \%$, have a characteristic morphology thus providing a detection criterion holding significance equal to that provided by the elemental composition. In fact, the particles must have a spherical or spheroidal shape and their surface should appear amorphous, devoid of any crystalline characteristic.

The diffractometric analyzes of GSR particles showed however the existence of a crystal lattice, evidently characterized by very small elements not detectable with the SEM.

This feature, combined with the formation mechanism determined by Basu, has led us to propose here an hypothesis related to the grain refinement phenomenon: the particles in the liquid state, formed during the primer deflagration, having gone through the gunpowder charge igniting it, undergo in the barrel an abrupt negative change of temperature. As a result of this rapid cooling, the grain refinement phenomena cause a dimensional reduction of the condensing crystalline species of at least two orders of magnitude.
\end{abstract}

Keywords: Gunshot residues; Spheroidal morphology; Nucleation and growth; Grain refinement

\section{Introduction}

The first known theoretical attempt to describe the formation of inorganic gunshot residues (GSR) was proposed by Basu [1]. He identified three categories of particles with spherical morphology and studied their origin and development during the cartridge discharge. A distinctive characterization of all three types of particles is that all have amorphous surfaces at the magnifications he used, and we still use, in the scanning electron microscope (SEM). Wolten., et al. [2] noted that GSR particles did not present features suggestive of crystalline structure configuration. But despite the fact GSR did not appear visibly crystalline, investigations by the same authors ascertained that GSR do have a micro crystalline structure (op.cit. page 18 Gunshot residue is not visibly crystalline although diffraction methods [ $x$-ray or electron diffraction] do give crystal patterns).

All subsequent studies, usually of a practical nature for casework application, have focused on the analytical and morphological nature of GSR formation without dealing with formation mechanisms and without attempting to explain the amorphous surface appearance.

Morin [3] has shown that a recent study by Chang and Williams [4] confirms the hypothesis of Basu [1].

The seminal work of Wolten and colleagues at the Aerospace Corporation in the United States was later developed by several authorities, notably by Andrasko, Nesbitt, Matricardi and Basu [2]. In England the Forensic Science Service - rightly regarded as the best specialized structure in the world and now sadly dismantled for financial reasons by a short-sighted government - has been at the top for research work from 1978 to its unwise dismantling some years ago. Talented scientists such as Keeley, Wallace and Mc Quillan (actually of the Belfast NIFSL) have refined the evaluation criteria that must be applied to the basic results provided by the SEM/EDX system. In Israel Tassa, Zeldes, Levin and Zeichner (almost all working in the Israel Police crime lab) have contributed significantly to our understanding of the formation and quantification of GSR. In Italy the first work on the GSR, more informative than scientific, was presented in 1981 at the L'Aquila (Italy) Congress of Forensic Ballistics [5]; after several years from this country 
arrived at an important contribution concerning GSR-like particles not deriving from firearms shooting. In fact, we had to wait until 1998 when Gentile and Morin, on the basis of personal communication obtained from Keeley, reported to the international scientific community that particles identical to gunshot residues could be formed by several varieties of automotive disc brakes. Two years later Torre (Turin University) published a significant work [6] on the same issue.

More recently Burnett (Meixa Tech, California, USA), Brożek-Mucha (Institute of Forensic Research, Krakow, Poland) and Gunaratnam (National Bureau of Investigation, Crime Laboratory, Finland) have contributed to a better understanding of GSR: in particular, Brożek-Mucha, with her numerous works on chemical and morphological properties of GSR particles, .has offered very remarkable contributions on the morphology topic.

Finally, the ever present question concerning the importance of the GSR particles morphology was discussed by a small community (IAMA NEWSLETTER 09-28-01, Volume 2, Issue 2, page 10) with the following paper How do you weight morphology in the interpretation of your P-GSR results?

The hypothesis we wish to propose is the following one: at the time of the solidification of the liquid particles (molten metal) formed by the primer mix explosion, mechanisms related to the phenomenon known as grain refinement decreases the size of the crystals of (about) two orders of magnitude so that the surfaces of the particles would appear amorphous, at least at the usual magnifications obtainable with the SEM.

\section{Materials and Methods}

The detailed analysis presented in this work has for object the relevant literature. The methods utilized are primarily theoretical instruments applied to the specific topics that will be discussed with this approach.

\section{The primer initiation}

The cartridge primer explosion is initiated by different mechanisms of energy transfer which originates with the crushing of the primer mixture between the capsule and the anvil produced by the firearm firing pin. The primer explosion initiates with two concurring phenomena: friction and thermal shock. Small bubbles of gas entrapped in the primer mass are subject to compression by the firing pin strike. This generates hot spots that give origin to the thermal ignition of the explosive primer mixture [7]. The hot spots are areas of small volume $\left(\approx 10^{-3}\right.$ to $\left.10^{-5} \mathrm{~cm}^{3}\right)$ in which an elevated amount of heat is generated in three major ways:

- Adiabatic compression of entrapped gaseous bubbles;

- Friction between explosive and retaining walls; intercrystalline friction between the particles of the explosive mixture. In some cartridges, e.g., 22 caliber rim fire cartridges, between the explosive and grit (usually silica or glass but also particles of antimony sulphide which also acts as a fuel)

-Viscous heating caused by the rapid movement of the explosive when squeezed between the impact surfaces.

The mechanism of adiabatic compression of small gas bubbles trapped in the mass of mixture, along with the one linked to friction, seems by far to be the preeminent. For the first mechanism, the compression ratio for the determination of the hot spots temperature, under the theory of perfect gases, can be determined through the relation

$$
\mathbf{T}_{\text {final }}=\mathbf{T}_{\text {initial }}\left(\mathbf{P}_{\text {final }} / \mathbf{P}_{\text {initial }}\right)[(\gamma-1) / \gamma]
$$

where $\gamma=\mathrm{Cp} / \mathrm{Cv}$ and $\mathrm{Cp}($ resp. $\mathrm{Cv}$ ) is the specific heat in constant pressure (resp. volume).

Bydal [8] notes: "Frictionators presumably provide additional heat energy when adjacent crystals of tetrazene (4-guanyl-1(nitrosoaminoguanyl)-1-tetrazene, an explosive compound sensitive to friction frequently present in the most common primer mixes) [9] are scraped during the rapid crushing process. Bowden and Yoffe [10] did a lot of careful experiments to demonstrate the high temperature produced in tiny areas of material during friction process. They showed how the melting point of the frictionatotors was important".

A recent study [11] determined, in a mechanics of materials point of view, a micromechanical model that leads to the formation of hot spots in the crystals of energetic materials that have been subject to impact.

The following Figure 1, present in 11, do not require any remark.

Furthermore, it should be emphasized that there is an intimate relationship between the porosity of high explosives, the impact sensitivity and the sudden heating [12]. It is worth remembering the fact that every reactive material (though admittedly not necessary energetic), pure or in mixture, can be initiated thermally when impacted [13]. For impact it is defined the lower limit of mechanic impulse required to start the mix initiation.

In order to provide a fairly complete review of these initiation mechanisms we have to reconsider the work of Afanas'ev and Bobolev [14] and Kholevo [15] who, in 1946, remarked that the inconsistency of the physical mechanisms of non-thermal initiation, where the heating mechanism due to impact that would develop in a rapid inelastic deformation of the energetic material, is emphasized as promising from theoretical and experimental point of view. Taking this into account, in the first stage of the impact, thanks to the deformation of single particles and the friction between them, their edges melt forming a viscoplastic biphasic system with 
liquid layers heated to high temperatures creating hot spots. Wenograd [16] reported the temporal development of the energetic phenomenon of high energy explosives when subjected to a sudden rise of temperatur. The delay time $\tau$ is provided by the following expression

$$
\tau=A \exp \left(\frac{B}{R T}\right)
$$

In which $\mathrm{T}$ is the absolute temperature of the test tube at the moment of the explosion, $\mathrm{A}$ and $\mathrm{B}$ are constants depending on materials and $\mathrm{R}$ is the gas constant.

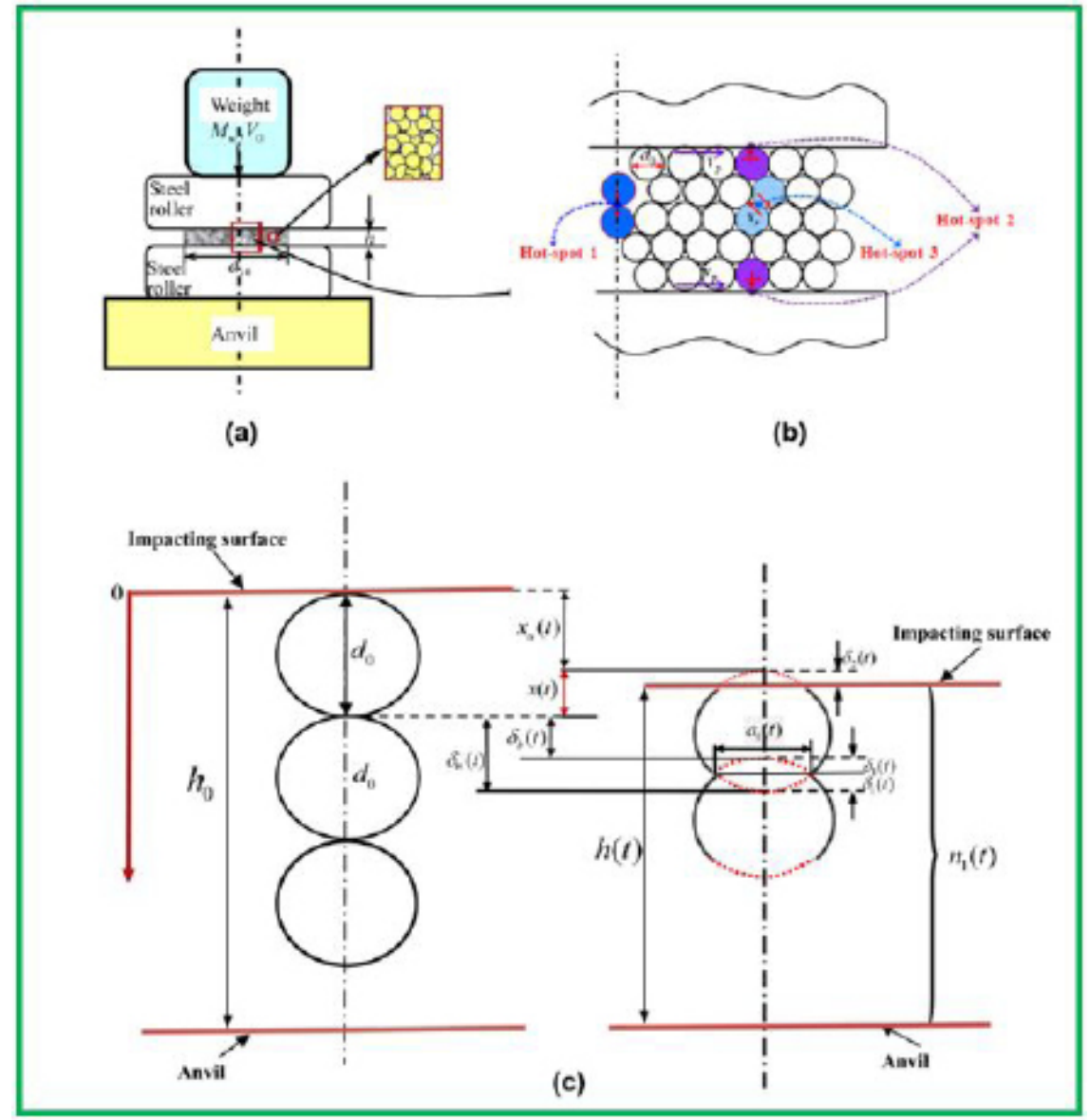

Figure 1: Microscopic model for hot-spot ignition of granular energetic crystals.

In a), b) and c) are represented, at various magnifications, the mechanic interactions between single crystal under crushing phenomena

Consider, for example, the composition (in percentages by mass) of No. 41 primer used for several decades in the M855 ammunition cal. 5,56 mm:

lead styphnate (37\%) - barium nitrate (32\%) - antimony sulphide (15\%) -

micro-powder aluminium $(0.7 \%)$ - tetrazene $(0.4 \%)$ - PETN $(0.5 \%)$

For each ingredient of the primer mix we can associate the following role:

$\checkmark$ Lead styphnate - initiator i.e. the explosive that, with the help of tetrazene, is activated with the percussion

$\checkmark$ Barium nitrate - oxidant i.e. provides oxygen for both the initiation and the combustion (it is a good oxidizing agent giving off oxygen at temperatures between $200^{\circ} \mathrm{C}$ and $300^{\circ} \mathrm{C}$ )

$\checkmark$ Antimony sulphide - the fuel that supports the primer flame (its crystalline nature makes it also a frictionator) [17]

$\rightarrow$ Aluminium micro-powder - raises the primer flame temperature

$\rightarrow$ Tetrazene - a sensitizer which works in conjunction with lead styphnate

$\triangle$ PETN - a high explosive that increases the mix brisance

The solid energetic primer material degrades when subject to liquefaction, evaporation or sublimation and / or decomposition. The presence of all or a part of these degradation types is dependent on both the energetic material and the ambient pressure.

In solid energetic materials characterized by medium-high vapour tension the sublimation or evaporation process will develop in the gas phase. The opposite will occur for energetic materials characterized by high melting points and low values of vapour tension: for this material the degradation will start in the condensed phase (liquid and / or solid). 
It is worth noting that for blasting explosives an extreme phenomenon characterizing the reaction is the formation of plasma in the course of the detonation. This detail is important to understand the behaviour to firearm primer initiation. The plasma generated by the detonation and characterized by the electrical properties of high ionization, was initially noticed on the exposed surfaces of high explosives. This apparently anomalous behaviour resulted impossible to associate with the thermal ionization of the shock wave propagating in the surrounding gaseous medium. It is clear that the plasma induces the initiation of the energetic materials which, in turn, causes the high ionization as a result of molecular dissociation and excitation of the electronic status. This condition is followed by collisional quenching.

Generally these effects together or separately can occur with the interaction of the plasma with gas and are of a dual nature: - Thermal, whereby the heating of the gas due to the release of energy leads to higher chemical kinetics while the uneven heating of gas volumes generates disturbances in flow, causing turbulence (and favouring, therefore, mixing).

- Non-thermal, due to the effect of the ionic wind (the drift of ions and electrons) and subsequent excitation, followed by the gas dissociation and ionization, due to the electronic impact, which leads to non-equilibrium states and to the production of radicals that influence the combustion kinetics.

It can be deduced that the active particles of the plasma produced in the primary detonation directly interact with the remains of the priming mixture mass and with the gas pockets trapped inside the primer material, given that:

- The nature of the ionized gas, like plasma, is neutral and consists of sets of electrons and ions. Higher the atomic number of the inorganic species detonating, the greater will be the production of electrons and ions torn from the respective atoms.

- The overall motions of charged particles in the plasma, due to the long-range electrical forces, characterize a electrically neutral but locally ambipolar state.

- Outside of the Debye spatial sphere, the potential energy is inferior to the kinetic energy of the particles resulting in free diffusion. - The dominant mechanism of the effect of primer initiation, induced by plasma, is a function of the generation of active particles in the plasma itself.

- In the case of gas trapped in the priming mixture, the most sensitive of atomic species is oxygen followed to a lesser degree by nitrogen and then by neutral radicals.

However, the priming mixture will be initially in solid phase (i.e., a condensed phase). It is impossible to understand the simultaneous degradation of the individual compounds with the mutual interaction of these products. We must deal with this problem by seeking a synoptic representation of the variables involved with the primer detonation.

Charles and Kerry [18] investigated the thermal and oxidative behaviour of a series of antimony sulphides using thermogravimetric analysis, differential scanning calorimetry, high temperature differential thermal analysis, and Fourier transform infrared spectroscopy. Antimony trisulphide was found thermally stable to $575^{\circ} \mathrm{C}$ and oxidatively to $300{ }^{\circ} \mathrm{C}$, above which it forms cubic $\mathrm{Sb}_{2} \mathrm{O}_{3}$. At temperatures above $600{ }^{\circ} \mathrm{C}, \mathrm{Sb}_{2} \mathrm{O}_{4}$ is formed. Antimony trisulphide undergoes a thermal reaction to produce $\mathrm{Sb}_{2} \mathrm{~S}_{3}$ between $195^{\circ} \mathrm{C}$ and $230{ }^{\circ} \mathrm{C}$. Antimony pentasulphide (containing 15 percent excess sulphur) undergoes a thermal transformation to yield $\mathrm{Sb}_{2} \mathrm{~S}_{3}$ between $193{ }^{\circ} \mathrm{C}$ and $239^{\circ} \mathrm{C}$. Removal of excess sulphur by extraction has little effect of the thermal or oxidative properties.

The fundamental variables of relevance in decomposition, combustion and detonation of energetic materials are time, pressure and temperature. The initial explosive phenomenon is detonative where detonation is defined as a combustion process with initial and very rapid propagation of a pressure wave. For this reason the primal gas undergoes a compression passing from the condition of reagent to that of products whereby the pressure wave generates the combustion wave. The coupled pressure and combustion waves, reach a speed in the order of several $\mathrm{km} / \mathrm{s}$ which is in contrast to the conditions of the unburned gases ahead of the flame front which have a lower speed.

In connection with high velocity of detonating wave propagation - $\mathrm{km} / \mathrm{s}$ - we can regard the phenomenon of detonation as a nanosecond process: which may be referred to as a stationary detonation. By virtue of the constraint imposed by the conservation of mass, momentum and total enthalpy, these gases are subjected to densification and to deceleration. The triggering mechanism, caused by a violent shock wave, causes a compression and the consequent rise in temperature, which, if sufficiently high, can lead to the ignition of the mixture. Furthermore, if the oxidation kinetics in this area is sufficiently fast, the result is that the expansion of gas can create a train of compression waves which enhance the first shock wave.

From this trigger mechanism derives the model of the one-dimensional detonation wave. Indeed, we can suppose that the detonation wave is formed of three contiguous parts. The first stage consists of a real shock wave, which spreads over a few molecular free paths. Therefore, after a few micrometers, a material which starts at atmospheric pressure undergoes a compression in the order of a factor of ten and the material temperature rises to values greater than that of autoignition. In the second stage the thermodynamic quantities remain unchanged for a volume that is directly proportional to the induction time of autoignition and detonation velocity. For particularly low induction times this stage may disappear, but generally it extends into the material for fractions of a millimetre. Finally, the third stage involves the portion of energetic material in which fast oxidation reactions take place. This is considered an authentic explosion with temperature rise, expansion and rarefaction of gases. To understand this evolution we need to refer to a Chapman-Jouguet (CJ) plot in which the values of pressure, density, temperature and velocity are taken in consideration. For example, in a simple case, when detonation occurs adjacent to a rigid wall, the detonated material will 
impinge against the wall resulting in an abrupt change in these variables. This spatial structure is the basis of the stationary onedimensional models proposed by different authors and which are referred to as ZND models, after Zeldovich, von Neumann and Döring, the three authors who have most contributed to the formalization of this type of models.

This model predicts a compression along the Rankine-Hugoniot curve without heat release, where the high pressure called the von Neumann spike (the explosive still remains unreacted). This transformation represents the first shock wave. The spike marks the onset of the zone of exothermic chemical reaction, which finishes at the Chapman-Jouguet state. After that, the detonation products expand backward. This transformation represents the first shock wave.

The greater approximation consists in the fact that a shock wave can not be described in detail by a Rankine-Hugoniot curve, because this is based on the balancing equations, while the shock wave requires a description of the gas kinetics as it involves only a few molecular free paths. The second stage cannot be described in the context of a pressure-density surface, because it corresponds to a total freezing of all the thermodynamic quantities. Finally, the third part is described as a material expansion that initiates from the pressure reached during the first compression. Several patterns are proposed depending on the model used. These models differ mainly in the proposed quantity of heat released or, equivalently, by the degree of advancement of the oxidation or by the final chemical composition. It is obvious that all the possible pressure-density final states must lie below the Rankine-Hugoniot curve which forecasts a final state of equilibrium. In the area of our interest, the time involved, essentially as the interval of time that elapses between a specific mechanical impulse and the achievement of pressures on the order of MPa and temperatures on the order of kilo ${ }^{\circ} \mathrm{C}$, lies in the order of microseconds.

The pressure and temperature values are, however, often opposing among them: indeed, the high temperatures usually favour reactions in which the products occupy volumes larger than the initial ones (with a consequential entropy increase), while high pressures, usually, favour reactions in which the volumes of activation are negative and/or subsequent products occupy a smaller volume. As we said, the degradation mechanisms of the solid phase takes into account, essentially, the phase changes of materials in different stages, the reactions between products, the nucleation and the growth of bubbles (liquid and/or gaseous). Furthermore, the high explosives porosity characteristics get involved in the critical small zones mechanisms of adiabatic heating, as occurs a transmission of compression waves and/or a percolation of hot gases.

In the range of temperature which increases from ambient to about $400{ }^{\circ} \mathrm{C}$, many of energetic materials are present as liquids or solids, so that:

$\checkmark$ In the liquid phase reactions occur in the proximity of the bubbles and the gaseous products may spread into the surrounding liquid

$\checkmark$ In the solid phase reactions occur on the particle surfaces interface with of nucleation and growth processes.

But the experimental evidence concerning primer detonation shows the sudden achievement of high temperatures in which the transfer and transport of material processes as described above would be interpreted as an intermediate step. The primer initiation is caused by the decomposition of lead styphnate, tetrazene and PETN. Analysis of the degradation mechanism from a thermodynamic point of view focuses first on the high primary explosives such as lead styphnate. Figure 2 is a graph of energy versus volume which represents the situation in which the primer is subjected to a mechanical impulse that submit the products in the non-reactive state (A) to a compression that shifts the energy-volume interrelationship to the Chapman-Jouget (CJ) point from which it starts an adiabatic evolution of the degraded products which culminates in their ambient air combustion (C). Specifically, the degradation products in adiabatic expansion release energy in the form of work PV and heat, while the turbulent and subsequent mixing of these products with the atmosphere's oxygen produces combustion with further release the energy.

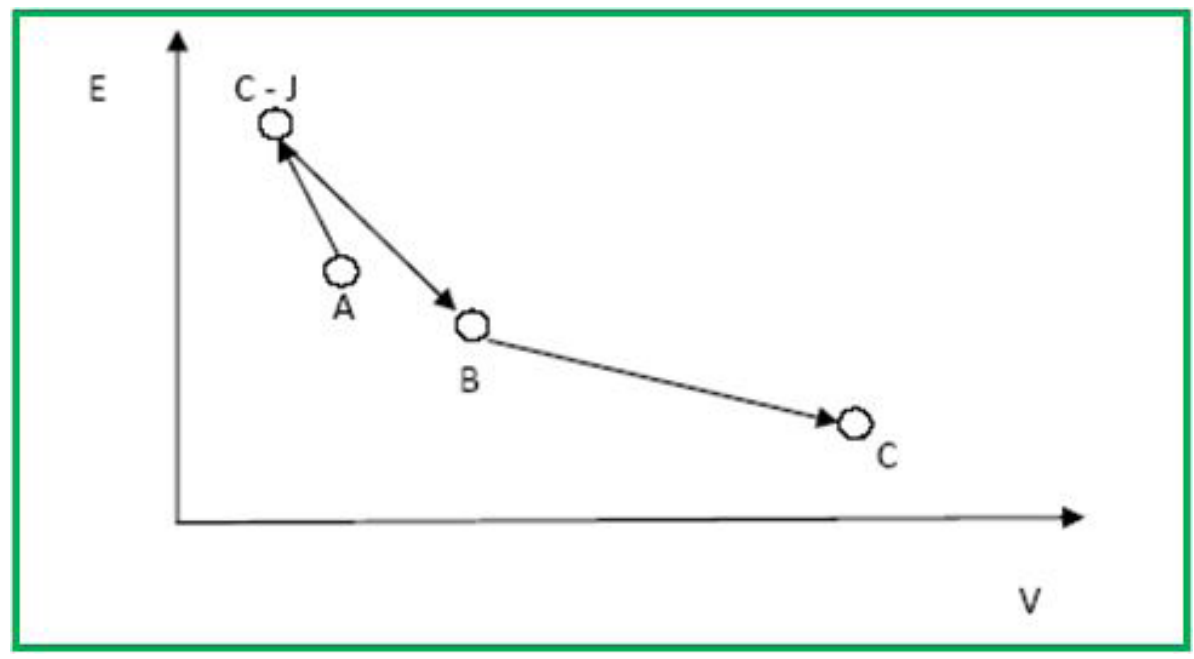

Figure 2: Evolution, in V (volume)- E(energy)representation, of energetic materials subject to impact 
In the primer the required oxygen is provided by the barium nitrate whereby the detonation event evolves until the degradation products combustion. It follows that only in the detonation phenomenon context; those typical values of temperature and pressure are achieved.

Some primer mixtures contain also aluminium powder which, in combination with lead styphnate and other primer components, develop a stationary detonating wave model of an heterogeneous charge (high explosive + metal particles of aluminium of ultra micrometer size scattered in the explosive) [19].

It has been found that there is a reduction in the detonation velocity in comparison with the corresponding value for the explosive (high potential) in its pure form or if assembled together to a mixture of inert particles. Furthermore, as it is represented in another study [20], the rate of aluminium combustion, in a non ideal explosive mixture, depends on the pressure and on the gases developed by the detonation products. The pressure and the detonation velocity of explosives containing aluminium powder are reported in another study [21] which configures the Becker-Kistiakosky-Wilson equations of $\mathrm{k}$ state parameter. In addition the study by Friedman [22] provides an excellent, elegant and complete thermodynamic approach to the detonation pressure prediction.

If we consider the detonation pressure $(\boldsymbol{p})$ effect, we note that it is affected by the density ratio: the detonation pressure is obtainable from the values of density $\rho_{0}$ (actual loading) and $\rho_{\max }$ (the theoretical maximum)

$$
p=p_{\max }\left(\frac{\rho_{o}}{\rho_{\max }}\right)^{2}
$$

where the pressures are measured in GPa, while the density in $\mathrm{g} / \mathrm{cm} 3$. This suggests, in agreement with Zhang and Chang [19], that for mixed explosives.

$$
p_{\max }=\sum\left(p_{e \max i} \frac{\alpha_{e v i}}{\rho_{e \max i}}\right) \rho_{\max }
$$

Nunziata and Morin warn the reader that in these equations from ref [19] pressure is presented as an extensive variable, (when, in fact, it is an intensive variable) like sum of the pressures of different sub-systems.

where $\mathrm{p}_{\max }$ in this case is the mixed explosives detonation pressure. $\mathrm{p}_{\text {emaxi }}$ is the theoretical detonation pressure of the $i^{\text {th }}$ species, $\alpha_{e v i}$ $=\mathrm{g}_{e i}\left(\rho_{\text {emax }} / \rho_{\text {emaxi }}\right)$ is the volume fraction of the ith species, $\mathrm{g}_{e i}$ is the mass fraction of the $i^{\text {th }}$ species, $\rho_{\text {emaxi }}$ is the theoretical density of the $i^{\text {th }}$ species.

By composing the two relations we obtain the formula for the detonation pressure of mixed explosives expressed in terms of mass fractions.

$$
p=\sum\left(p_{e \max i} \frac{g_{e i}}{\rho_{e \max i}}\right)\left(\frac{1}{\rho_{\max }}\right) \rho_{o}^{2}
$$

Relative to the initiation and propagation of primary explosives [23], the following phenomena are relevant:

1. Shock to detonation transition (SDT)

2. Deflagration to detonation transition (DDT)

3. Dead pressing (DP).

We have dealt previously with the first mechanism. The second mechanism (DDT) is implemented when deflagration take place (in the absence of pre-existing shock waves), while propagating in a granular explosive which accelerates the detonating of the same explosive. The mechanisms are multiple, competing between and not, but completely defined (thermal conduction laminar intrusion of hot gaseous products, compaction of the grains etc).

Dead pressing (DP) is the most interesting as it introduces us to primer mixture phenomenology: indeed DP is a problem associated with the granular compaction as well as the porosity of primary explosives and can be interpreted as a problem due to inefficient initial deflagration propagation in the mass of the explosive in such a way that the detonation doesn't occur.

In a summarizing the primary explosives proprieties [24], we need to consider in the forthcoming arguments,

$\checkmark$ The influence of the loading density

$\checkmark$ The influence of the specific surface area

$\checkmark$ The influence of the diameter of the charge 
$\checkmark$ The influence of confinement

$\checkmark$ The influence of the presence of a secondary explosive

D The sensitivity (to shock, friction, electrostatic discharges, flame).

Lead styphnate, in its role as initiator, is phlegmatic when surrounded by other compounds. In fact, the lead styphnate in the primer cup is dispersed into other substances. In order to support the mechanism of initiation, the priming mix is doped with small amounts of high explosive [25].

The primer mix explosive can be defined, appropriately and without fear of contradiction, as a low brisance deflagration [26].

Matyas \& Pachman [27] stated "Priming compositions or priming mixtures are sensitive explosive mixtures that are designed to produce a flame in a particular application. They are most often mixtures containing a primary explosive as one of the components in such a form and amount that ensures the inability to initiate detonation of the mixture. The role of the primary explosive is to sensitize the mixture to external stimuli, not to make it detonatable. Priming mixtures are used in percussion caps for ignition of gun powder, on electric fuse heads, delay elements, etc".

In a Master of Science thesis from the Pennsylvania State University [28] it is stated that:

$\checkmark$ The time needed for total consumption of the primer mix can be estimated to be between $70 \mu$ s or $90 \mu$ s;

$\checkmark$ Primer detonation reached temperatures of $2700{ }^{\circ} \mathrm{K}$.

Kuo, Moore \& Ghent [29] provided temporal trends (Figure 3) of the pressure that developed in a confined location next to the flash hole (a control chamber incorporating a piezometric transducer and where condensed matter was collected) and established that, in such an environment, $\sim 40 \%$ of the combustion products were in the condensed phase.

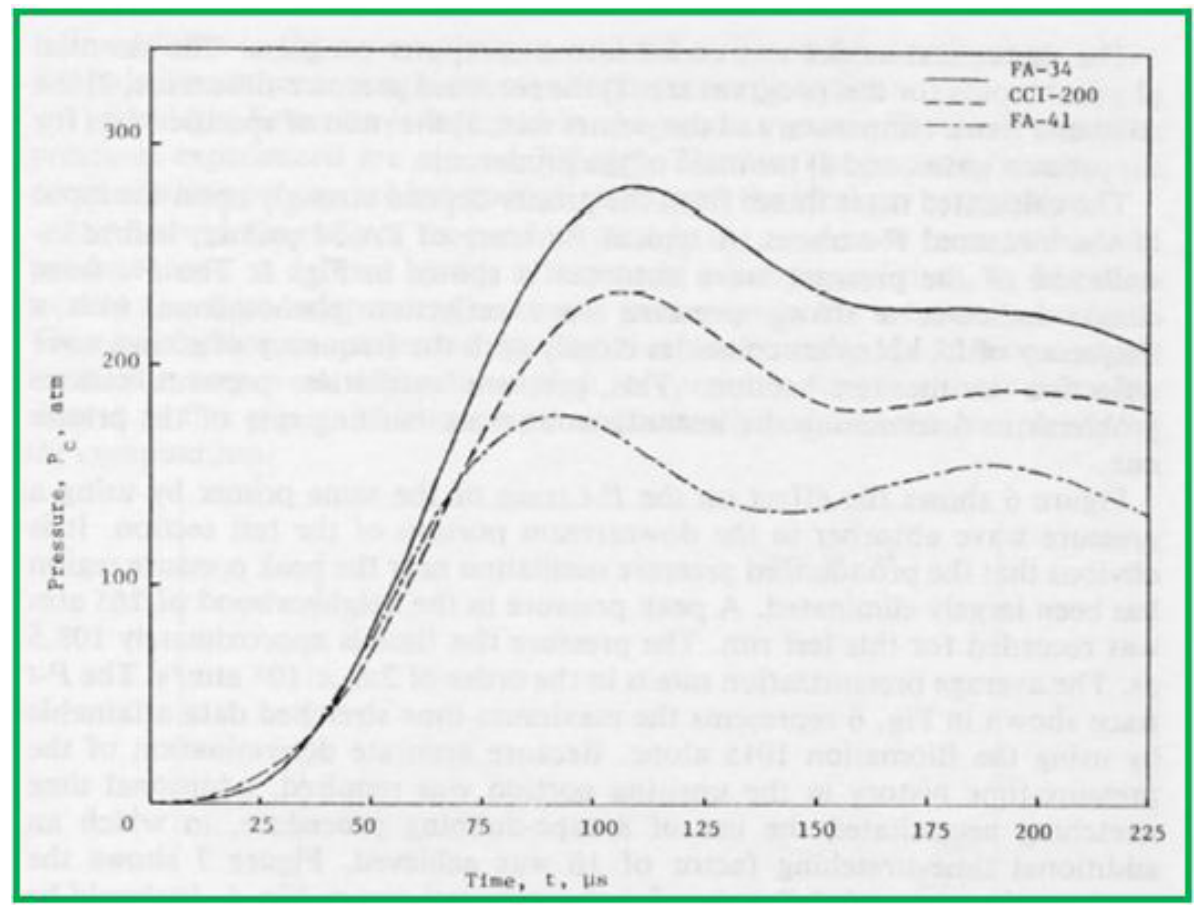

Figure 3: Comparison of time-stretched P-t traces for FA-41, FA-34 and CCI-200 primers

Furthermore, in the time required for the primer mix to combust, estimated to about 100 microseconds, pressures of about 300 Atm (30MPa) and temperatures of $2900^{\circ} \mathrm{K}$ were measured in the control chamber next the flash hole: please note that pressures of about $300 \mathrm{Atm}(30 \mathrm{MPa})$ isn't the pressures inside the pocket but this phenomenon is due to multiple wave reflections within the control chamber.

This pressure data agrees well with a study sponsored by the US Air Force Academy which noted that in the muzzle of a long barreled weapon pressures of the order of $1,5 \mathrm{MPa}-3,8 \mathrm{MPa}$ are reached.

It can be inferred as criterion plausibility that within the small volume of the primer cup, the decomposition products form a supersaturated environment in which there would be condensation to the liquid state. This hypothesis does not appear contradictory with other studies $[23,24,26,30]$.

In relation to the values of $3000{ }^{\circ} \mathrm{K}$ and $30 \mathrm{MPa}$ (i.e. those found in named control volume), the individual components of the primer mixture inside the confined volume of the cap could exist part of the time during the reactive process, perhaps in a dualphase liquid/vapour. 
Generally, the total energy provided by a energetic material may be estimated by analyzing the reaction mechanisms that occur in two different modes after the initiation. A primary reaction occurs between the oxidant and the fuel i.e. the oxidant decomposes increasing the average temperature of the material itself, as the free oxygen released in gaseous state that reacts with the fuel generating heat. A subsequent secondary reaction is between the unreacted and partially reacted fuel with oxygen in the atmosphere. This secondary reaction is described as "after burn" and, generally, produces a quantity of heat up to three times that released by the primary reaction.

If the energetic material is a pure primary explosive, then a main detonation type reaction is obtained which is independent of the environment's chemical condition and which reaches completion in the order of microseconds. On the other hand, the after burn of the primary detonation products in air (deflagration type) may last milliseconds. Because of this relatively long time interval, the secondary reaction is capable of producing an increased wave front of the primary detonation. The effects of the post-combustion increases in a condition of oxygen deficiency and when the energetic material is structurally oxygen poor.

Conversely, if the materials are less reactive, then the primary reaction proceeds as a deflagration with a less vigorous wave front. However, the effects of the subsequent secondary reaction are influenced if the energetic material is confined in a totally or partially closed setting. Obviously, these net distinctions are not always valid in the case of a priming mixes due to the wide variety of the explosive properties of the different components.

The thermo-chemical evaluation of the individual energetic materials present in the priming mixes is complex. The main factor is the energy produced in the decomposition and/or combustion process. In this sense, there are indications that the decomposition energy is linked to sensitivity and therefore to the initiation of the compound $[31,32]$.

Important related information may, therefore, be derived from the estimate of the heat of formation $\Delta \mathrm{H}_{\text {form }}$.

For the energetic materials under examination, during the time range involved, the physical state can be solid or liquid whereby, for the gaseous phase:

$$
\begin{gathered}
\Delta \mathrm{H}_{\text {form(liquid) }}=\Delta \mathrm{H}_{\text {form(gas) }}-\Delta \mathrm{H}_{\text {vap }} \\
\Delta \mathrm{H}_{\text {form(solid) }}=\Delta \mathrm{H}_{\text {form(gas) }}-\Delta \mathrm{H}_{\text {sub }}
\end{gathered}
$$

where $\Delta \mathbf{H}_{\text {sub }}$ represents the heat of sublimation while $\Delta \mathbf{H}_{\text {vap }}$ represents the heat of vaporization.

In this sense, the Le Chatelier diagrams supported by appropriate computer codes may shed light on the main aspects of these issues, but this approach is beyond the scope of the work presented here. The apparatus used in previous studies are vented bombs. Specially designed calorimetric bombs used in a number of studies $[23,24,26,29]$ to measure the heat generated by these processes allow the gases to escape from an orifice during the primer degradation, minimizing the loss of heat due to conduction within the given time intervals involved. This circumstance allows the reliable measurement of thermodynamic parameters such as pressure and temperature. For obvious physical reasons these values represent the lower estimate of these parameters that occur inside the primer chamber.

Finally, the initial escape of gases from the primer vent determines a better use of the internal volume by the remaining mix degradation products, whereby the latter average temperature decreases.

Bauer, Cook and Keyes [33] observed luminosity phenomena when high explosives were detonated and interpreted this as caused by diluted plasma in which external electron have been removed from both atoms and molecules. Peripheral valence electrons would have to be promoted to a collective condition allowing them to move freely but with enough negative potential energy to produce cohesion inside the plasma.

The evanescent behaviour of these plasmas could play a role for those primary energetic materials that contain inorganic elements, such as primers mixtures. Indeed, it is plausible to hypothesize the presence of additional free atomic and/or molecular ions that would promote chemical recombination. The metastable matter escapes through the flame vent as a gas jet, which, due to the increase in volume within the cartridge case and thermal jump, leads to the formation of liquid and solid masses. The superheated saturated steam which is at a temperature higher than that corresponding to the vaporization temperature, suddenly cools to a temperature below the condensation temperature, producing other droplets.

Chang and Williams [34] reported the presence of particulates, in a condensed form (liquid and/or solid), in the flow of gases from the flame vent. The presence of particulates was confirmed by a subsequent experimental stage: in fact the distinction between the presence of particles and their absence is due to a different ignition response of the propellant since the presence of particles has resulted from a more efficient powder ignition. 
Given this process, the detection of particles inside the primer cup can be attributed to:

$\checkmark$ The backwash of particles from the shell powder chamber

$\checkmark$ The relative rapid decrease of the primer due to the thin capsule contact with the exterior

$\checkmark$ The heterogeneous nucleation (see below) especially due to anvil curved geometry [35].

In this we disagree with Basu's interpretation, schematically represented in Figure 4.

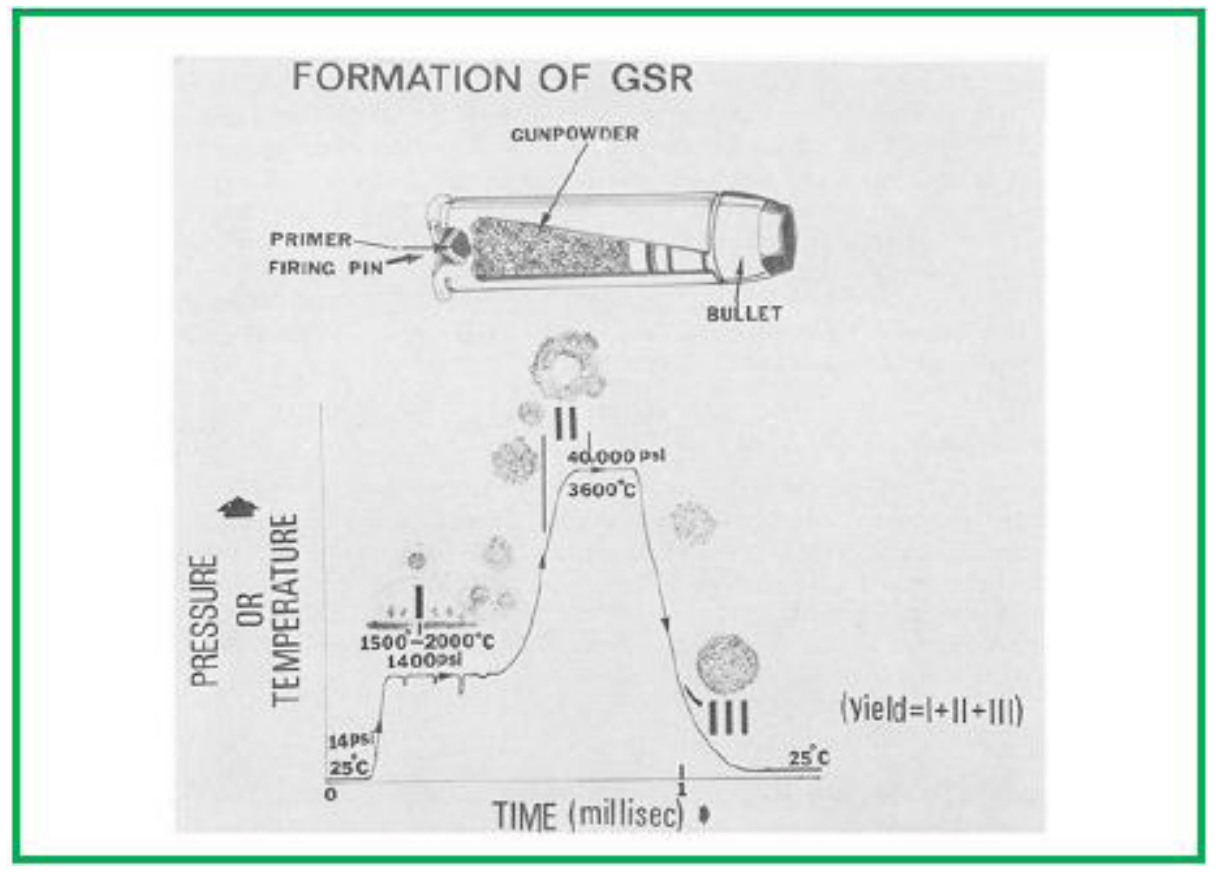

Figure 4: The formation of gsr via three stages (I, II and III) of primer explosions (1psi = 6,89 kPa)

Morin [3] has highlighted a correction to Basu's estimated pressure and temperature values in the inside the primer cup: indeed, the pressures are lower than the average specified, while the temperatures are higher than the values theorized by the Author.

We should remember that S.E. Stephenson [36] settled the maximum recorded pressure as never exceeding $2 \mathrm{MPa}$ (and not at the order of 9,65MPa from Basu hypothesis).

At this point we must ask what are the conditions that drive the phase change in the liquid $\rightarrow$ solid transition. The stages after the bullet leaves the barrel will be characterized by a thermal and pressure balance with the outside environment, which induce a local undercooling conditions.

Basu's work can be reinterpreted in the context of the grain refinement hypothesis. This hypothesis would agree with the fact that the particles that Basu postulates as forming in "STAGE I", namely those formed in the first moments of the primer initiation and which are of a small size, are most influenced by the effects of grain refinement.

This assertion relating to the vapour-liquid transition and the effects of grain refinement are supported by the findings of Buckle and colleagues [37] who establish that if metal-particle nucleation occurs from the aerosol phase in the presence of a significant thermal jump, it will result in regular spheroidal particles.

\section{Interpretation in the context of the classical nucleation theory (CNT)}

At this point, to guide the reader, it's necessary to remind some knowledge of thermodynamics. Generally, the free energy change between the initial and final stage measures the tendency of an isothermal and isobaric process to unfold spontaneously: it is thermodynamically disadvantageous if $\Delta \mathbf{G}>0$, but if $\Delta \mathbf{G}<0$ the process is favoured and tends to take place spontaneously. In equilibrium (in the strict sense), the system is characterized by $\Delta \mathbf{G}=\mathbf{\Delta} \mathbf{H}-\mathrm{T} \boldsymbol{\Delta} \mathbf{S}=0$ (where $\mathrm{G}=\mathrm{Gibbs}$ function, $\mathrm{H}=$ enthalpy, $\mathrm{S}$ $=$ entropy).

The liquid $(\mathrm{l}) \rightarrow$ solid (s) transition (and vice versa) occurs at a precise solidification temperature denoted as Ts (or melting temperature $\mathrm{Tm}$ ). Above this temperature the liquid phase is stable and below, always under thermodynamic equilibrium conditions, is stable for the solid one. In our case, considering the first moments involving the GSR formation phenomena, we consider as reasonable the process as isothermal and isobaric [38]. Referring to the Gibbs function (G= H - TS), the temperature $\mathrm{Tm}=$ Ts shows that $\mathrm{Gl}=\mathrm{Gs}$ i.e. $\Delta \mathrm{Gls}=\mathrm{Gs}-\mathrm{Gl}=0$. The liquid $\rightarrow$ solid step involves the formation of a more ordered set of atoms, which corresponds to a decrease in entropy, $\Delta \mathrm{Sls}=\mathrm{Ss}-\mathrm{Sl}<0$ (a negative change of entropy corresponds to an output of heat by 
the system, which is equal to the so-called latent heat of solidification LS). The condition of no variation of G implies that imposing $\Delta \mathrm{Gls}=\Delta \mathrm{Hl}-\mathrm{T} \Delta \mathrm{Sls}=0$, it must necessarily be $\Delta \mathrm{Hls}<0$.

In the liquid $\rightarrow$ solid phase the atoms are aligned in lattices ordered with generally increasing density. In real conditions - those approaching thermal equilibrium with the external environment- it will be possible, though, to have a confined spatial situations characterized by disequilibrium, with undercooled liquid below the temperature Tm, for which $\Delta \mathrm{Gls}=\mathrm{Gs}$ - Gl $<0$ (thermodynamically favoured process). This condition may occur in the inner zones of the cartridge case which, for obvious reasons, are characterized by a very localized chaotic evolution induced by particular boundary conditions (adjacency between the cartridge chamber and the outer wall of the cartridge case, the thermal capacity of the latter considered as a reservoir at T temperature). During solidification, the atomic aggregation varies from short-distance ordered structures to long distance. Solidification occurs due to nucleation (the formation of a solid form with particular characteristics) and to the accretion (entities of liquid binding themselves to the solid part while giving away vibrational energy) $[39,40,41,42]$. The priming mixes generally consist of $\mathrm{Pb}, \mathrm{Ba}$ and $\mathrm{Sb}$ inorganic salts, as shall later discuss, when activated develop molten metal liquid $\rightarrow$ solid phase transition. A metal generally solidifies when its liquid phase cools to just below its solidification temperature. This is because the energy associated with the crystalline structure is less than to that of the liquid phase. Within the framework of the classical nucleation theory (CTN) the difference in energy between the solid/crystalline and liquid states is $\Delta \mathbf{G}_{\mathrm{v}}$, the free energy associated with the volume and while the aggregate solid grows in size, $\Delta \mathbf{G}_{\mathbf{v}}$ decreases. During the formation of a solid aggregate (Figure 5) an interface is generated between it and the surrounding liquid. This interface is associated with free energy per unit of surface $\sigma$ proportional to the solid in formation size $[43,44,45,46,47,48]$. The total change in free energy $\Delta \mathbf{G}$ is equal to

$$
\Delta G=-\pi r^{3} \Delta G+\pi r^{2} \sigma
$$

where $\frac{4}{3} \pi r^{3}$ is the volume of a spherical embryo of radius $r$ while $4 \pi r^{2}$ is its surface, $\sigma$ is the free energy per unit of surface and finally $\Delta \mathbf{G}_{\mathbf{v}}$ is the free energy per unit of volume

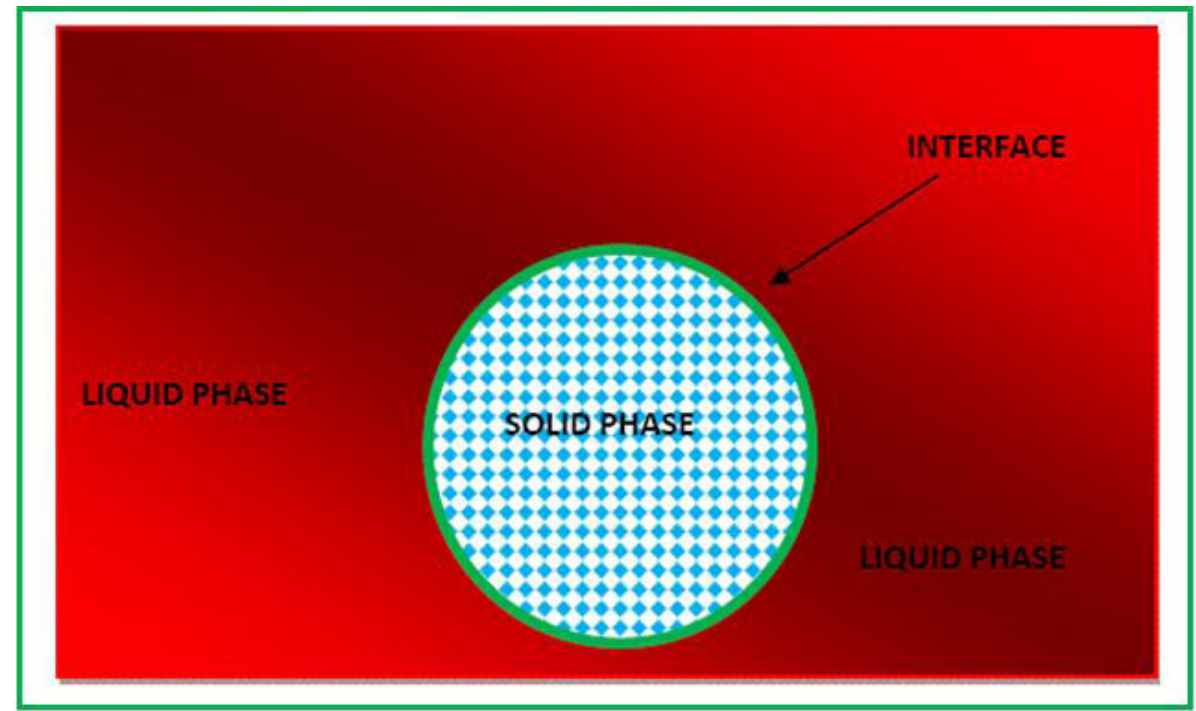

Figure 5: Schematic formation of a solid aggregate

When the solid aggregate is very small, a further size increase causes an increase in free energy. In such metastable conditions instead of growing solid the system will liquefy again, since there is a decrease in free energy, a favourable thermodynamics condition. This small solid aggregate is called "embryo" and as a liquid is below its solidification temperature, this is a undercooled condition.

The undercooling temperature $\Delta \mathrm{T}$ is the solidification temperature (at equilibrium) minus the temperature of liquid.

However when the solid has a radius greater than a particular value of $\mathbf{r}^{\star}$, accretion cause a decrease of the total energy; the solid aggregate that forms is now stable and we have the nucleation and accretion of the solid particle (the nucleus) (Figure 6).

The total free energy of the solid-liquid system, which is in development, changes with the magnitude of the solid aggregate size. The aggregate is a solid embryo if its radius is smaller than the critical radius $\mathrm{r}^{\star}$ and is a nucleus if its radius is larger than the same critical radius $\mathbf{r}^{*}$. 


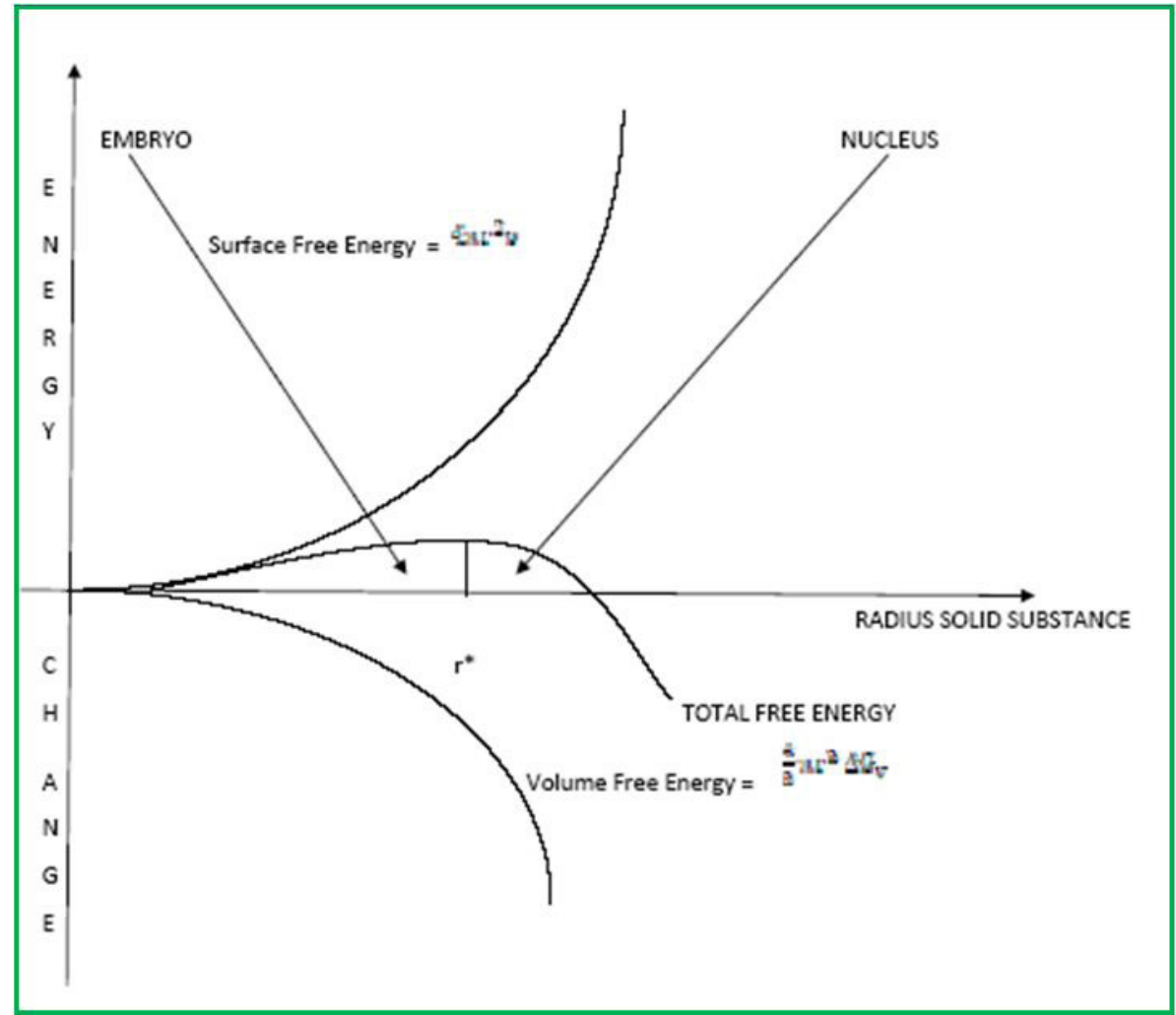

Figure 6: Synoptic chart (Volume- Surface - Total) free energy versus radius of material in development

When the liquid phase is cooled to below the solidification temperature, two competing factors combine to promote nucleation: the clustering of atoms (forming large embryos) and, if there is a free energy difference between the solid and the liquid phase, a reduction of the nuclei critical size occurs. The formation of a stable nucleus depends on the competition between the forces driving the phase change from liquid to solid (free energy per unit volume) and the energy required for the formation of a new interface. A homogeneous nucleation occurs when, starting from the above conditions, the undercooling becomes large enough so as to form the stable nuclei.

$$
\text { The critical radius will be equal } r^{*}=\frac{2 \sigma T_{m}}{\Delta H_{f} \Delta T}
$$

where $\Delta \mathbf{H}_{\mathrm{f}}$ is the latent heat of fusion (the heat released during the transformation liquid $\rightarrow$ solid), $\mathrm{T}_{\mathrm{m}}$ the melting temperature, $\Delta \mathrm{T}$ $=\mathbf{T}_{\mathrm{m}}-\mathbf{T}$, undercooling temperature $\mathrm{T}$.

Therefore, as the undercooling increases, the radius required for nucleation decreases.

Finally, the work needed for the formation of a spherical nucleus is given by

$$
\mathrm{w}=\frac{16 \pi \sigma^{3}}{3 \Delta \mathrm{G}_{\mathrm{v}}^{2}}
$$

A radius of curvature, $r$, bigger than the critical radius $\left(\mathbf{r}^{\star}\right)$ is obtainable with a small surface area, for example interposed between solid and liquid. It only takes a few atoms aggregated together to promote the growing particle to the critical size (so that the nucleation occurs more quickly). The nucleation generated on the surface of a particle of debris is called heterogeneous nucleation: a nucleus that develops on a heterogeneous surface will tend to assume a critical radius. Indeed, the radius, $r$, of the hemisphere which incorporates the solid phase is inferior to the one determined by the hypothetical circular surface (shown by the dashed line). With a small increase of the surface free energy, heterogeneous nucleation can occur, usually with comparatively low undercooling.

Qian [49] proposed various models, shown in Figure 7, of grain formation for heterogeneous nucleation on different surface shapes.

Because the actual volume of the solid phase is a fraction of the volume that would be occupied if the nucleation was homogenous, it follows that the work necessary for the formation of the actual volume is reduced. The interface that forms is stabilized by surface tension effects of the nucleating substrate, for which the work, W, needed for the formation of a heterogeneous nucleation will then equal: 


$$
\mathrm{W}=\left[\frac{(2+\cos \theta)(1+\cos \theta)^{2}}{4}\right]\left[\frac{16 \pi \sigma^{3}}{3 \Delta \mathrm{G}_{\mathrm{v}}^{2}}\right]
$$

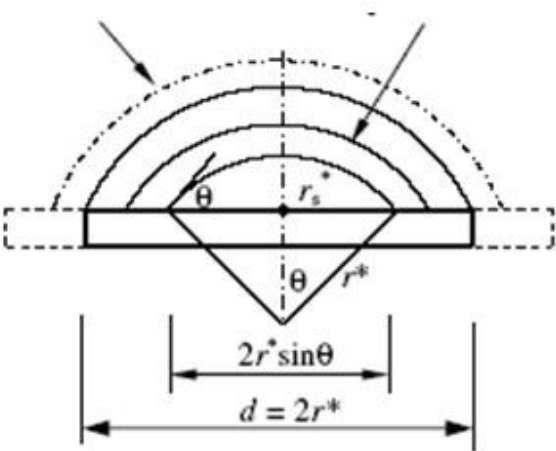

a

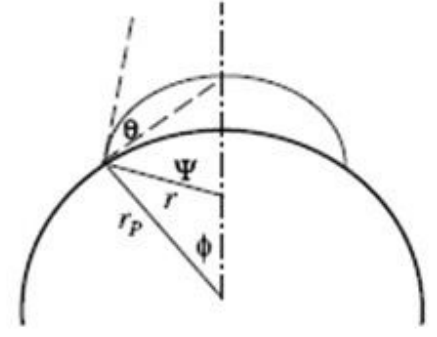

b

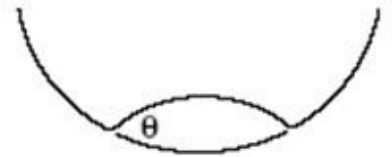

C

Figure 7: Various models of grains formation for heterogeneous nucleation on different surface shapes: a) flat b) convex c) concave (reproduced with permission of author)

Heterogeneous nucleation takes place in the saturated or supersaturated solution, which, through the mechanism of heterogeneous catalysis, actives weak bonds at the interface with the molecules in solution, promoting a crystallization reaction through the formation of crystal seeds. The activation energy decreases considerably if nucleation occurs on defects such as grain boundaries, voids, dislocations, the container surfaces and impurities. As soon as a nucleus forms, atoms in the solution accrete onto the newly formed solid object. The accretion modalities depend on the way in which heat dissipates. Heat from two sources must be removed from the system: heat generated by the latent heat of the liquid dissipates through irradiation and/or convection and heat generated by the latent heat of fusion must be subtracted from the solid-liquid interface to ensure complete solidification. The way in which the latent heat of fusion is subtracted determines the accretion mechanism and the final structure of the solid.

\section{Application of CNT to the formation of GSR}

The undercooling of the liquid metal below its melting point is a common feature of cast substances. The undercooling phenomenon influences the nucleation in the formation of both crystalline and amorphous formations. From the preceding discussion, Classical Nucleation Theory (CNT) can be applied in the isothermal and isobaric condition existing inside the primer cup during its deflagration. We assume, for simplicity, only homogenous nucleation.

In homogenous nucleation, by setting $\mathrm{d} \Delta \mathrm{G} / \mathrm{dr}=0$ in $\Delta \mathrm{G}=\frac{4}{3} \pi \mathrm{r}^{3} \Delta \mathrm{G}_{\mathrm{v}}+4 \pi \mathrm{r}^{2} \sigma$, the critical values, corresponding to the maximum, are obtained (denoted by superscript ${ }^{*}$ ) $\mathrm{W}=\Delta G^{*}=\frac{16}{3} \pi \frac{\sigma^{3}}{\Delta G_{v}^{2}}$ and $\mathrm{r}^{*}=\frac{2 \sigma \mathrm{T}_{\mathrm{m}}}{\Delta \mathrm{H}_{\mathrm{f}} \Delta \mathrm{T}}$. Free energy changes on nucleation are due to bulk free energy reduction + increase in surface energy +increase in strain energy. We recapitulate using the following picture Figure 8.

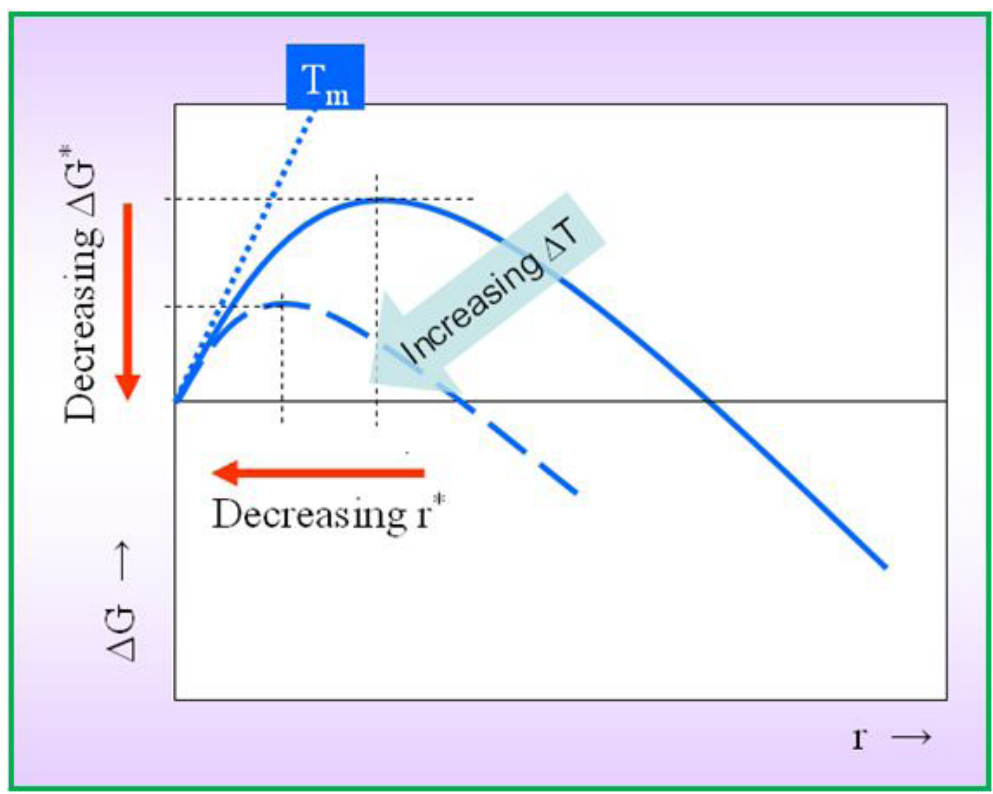

Figure 8: Phase Transformations in Metals and Alloys D. Porter - K. Esterling (Van Nostrand Reinhold Co. Ltd., New York - 1981) 
With this point of view, Turnbull's approximation $\left(\Delta \mathrm{G}^{*}=\frac{16}{3} \pi \sigma^{3} \frac{\mathrm{T}_{\mathrm{m}}^{2}}{\Delta T^{2} \Delta \mathrm{H}_{\mathrm{f}}^{2}}\right)$ is the most appropriate configuration to investigate the homogenous nucleation of primer particles. Consequently, the theoretical trend of critical radius is function of undercooling.

When a non-eutectic alloy solidifies, its components solidify at different temperatures, exhibiting a plastic melting range. Conversely, when a well-mixed, eutectic alloy melts, it does so at a single, precise temperature. However relatively to a tri elemental composition alloy we can infer that liquid droplets are eutectic alloys characterized by an eutectic temperature (the lowest possible melting temperature over all of the mixing ratios for the involved component species). If the liquid droplet of lead, barium and antimony has a eutectic point, then the melting point is lower in respect to a melting temperature of single elements. For this reason the effect of undercooling will be more evident. This system close to the eutectic could be susceptible to amorphization by rapid quenching in terms that shall be explained later.

For example, we can build the following table of the critical radius size for $\mathrm{Pb}$ according to the function of the thermal jumps.

\begin{tabular}{|c|c|c|}
\hline & & $\mathrm{Pb}$ \\
\hline latent heat of fusion & $\mathrm{Hf}\left(\mathrm{cal} \mathrm{g}^{-1}\right)$ & 5,9 \\
\hline latent heat of fusion & $\mathrm{Hf}\left(J g^{-4}\right)$ & 24,6856 \\
\hline volumetric mass density of liquid state & $\rho\left(\mathrm{g} \mathrm{cm}^{-3}\right)$ & 10,7 \\
\hline melting point & $T_{m}\left({ }^{\circ} \mathrm{K}\right)$ & 600 \\
\hline latent heat of fusion per unit volume & $\Delta \mathrm{Hf}\left(\mathrm{J} \mathrm{m}^{-3}\right)$ & 264,14 \\
\hline free energy per unit surface $@ \mathrm{~T}_{m}$ & $\sigma\left(J \mathrm{~m}^{-2}\right)$ & 0,0046 \\
\hline ambient temperature & $T\left({ }^{\circ} \mathrm{K}\right)$ & 323 \\
\hline undercooling $T_{m}-T$ & $\Delta T\left({ }^{\circ} \mathrm{K}\right)$ & 277 \\
\hline critical radius $r^{*}$ & $\mu \mathrm{m}$ & 75,44 \\
\hline
\end{tabular}

Table 1

\begin{tabular}{|c|c|c|}
\hline & & $\mathrm{Pb}$ \\
\hline latent heat of fusion & $\mathrm{Hf}\left(\mathrm{cal} \mathrm{g}^{-4}\right)$ & 5,9 \\
\hline latent heat of fusion & $\mathrm{Hf}\left(\mathrm{Jg}^{-2}\right)$ & 24,6856 \\
\hline volumetric mass density of liquid state & $\rho\left(\mathrm{g} \mathrm{cm}^{-3}\right)$ & 10,7 \\
\hline melting point & $T_{m}\left({ }^{\circ} \mathrm{K}\right)$ & 600 \\
\hline latent heat of fusion per unit volume & $\Delta H f\left(\mathrm{~m}^{-3}\right)$ & 264,14 \\
\hline free energy per unit surface $@ T_{m}$ & $\sigma\left(\mathrm{J} \mathrm{m}^{-2}\right)$ & 0,0046 \\
\hline ambient temperature & $\mathrm{T}\left({ }^{\circ} \mathrm{K}\right)$ & 290 \\
\hline undercooling $T_{m}-T$ & $\Delta T\left({ }^{\prime} K\right)$ & 310 \\
\hline critical radius $\mathbf{r}^{*}$ & $\mu \mathrm{m}$ & 67,41 \\
\hline
\end{tabular}

Table 2

However, in practice, spherical lead, barium and antimony primer GSR particles of less than $1 \mu$ m diameter are commonly encountered. The explanation for this disparity between theoretical calculations and the real-world observations cannot be found in the thermal gradient induced by the undercooling but may be explained by other circumstances, such as rapid solidification processing.

It should be noted that the undercooling temperatures of more than $200{ }^{\circ} \mathrm{K}$ have physical meaning for the crystallite nucleation under thermodynamic equilibrium which is the basis of the involved equations [50]. If a molten metal is undercooled to below its melting temperature, due to the excess Gibbs free energy, then it's not in a stable phase. In addition, as $\mathrm{N}_{2}$ is a major component of air and has high thermal conductivity, a strong under-cooling is assured.

Vice versa the primer fused particles can travel in the open space or collide with the walls of the cartridge case and of the firearm bore. A supplementary heterogeneous nucleation may be governed by the extensive undercooling on contact with the over mentioned walls of the cartridge case and firearm bore. To appreciate this phenomenon of sudden cooling, it is observed that on touching cartridge cases ejected from a semiautomatic pistol or the barrel of a revolver immediately after discharge, they are only slightly warm. Furthermore the presence of free $0_{2}$ induces surface oxidation of molten metals, which is also a possible source of heterogeneous nucleation. Since metal oxides are in many cases more thermodynamically stable compared to the base metal, they can act as heterogeneous nucleation sites and limit the temperature range of undercooling. 
What we have so far represented is functional to introduce the grain refinement mechanism as a possible explanation of the phenomena that accompany the formation of the GSR particles.

Now we represent the interpretation in the detailed evolution modality domain of the typical grain refinement. In fact in this case the phenomena more similar to those of events subsequent to the initiation of the priming mixes are those known as rapid quenching, falling into the category of rapid solidification processing (RSP) that occur with high cooling rates (typically ranging from $10^{4}{ }^{\circ} \mathrm{C} / \mathrm{sec}$ to $10^{8}{ }^{\circ} \mathrm{C} / \mathrm{sec}$ ). The development of completed structures during the RSP processes can be interpreted, locally, in terms of free energy availability concurrent to the non-equilibrium phase, in turn correlated to the undercooling degree: with increasing of this last, the evolutionary availabilities (competitive among them) of different microstructures will increase. This suggests that the local undercooling would play a key role more significant than the normal cooling rate.

Thus, it seems remarkable that the dispersion of the priming mixes initiation products are distributed in topographies that represent islands of undercooling.

The microstructures evolution during the RSP phenomena must also take account of the effects of intermediate coalescence, which determine rapid supplies of latent heats of fusion: in this case the overcooled microstructures (in formation) represent, in a first approximation, an adiabatic system that will tend to evolve, especially in relation to the mechanical perturbations suffered.

The nucleation of a solid from a liquid phase is a process that involves small clusters of atoms (of various sizes) formed by aggregation or by dissolution in the case of a heterogeneous nucleation: it will occur due to nucleating agents (distributed in the volume of liquid or on the interface) that will lead to a different response to local undercooling. In fact, effects of nucleating agents will affect size, morphology and composition of the grain. This because the elevation of crystalline phase to varying degrees of organization and regularity is determined by the value of the interface (between the nucleating agent and the liquid) free energy, the value of which has to be greater than the free energy denominating the configuration with the solid. If undercooled, a metal in the liquid state may solidify in grains, whose dimensions are a function of the temperature at which the process takes place and whose edges represent an important area of discontinuity of the metal structure [51,52]. During the solidification the atoms must be grouped in ordered sets and distinct from the liquid: this occurs much more readily the greater is the undercooling. Once the nuclei are formed, the growth may proceed with relative ease [53-55]. As stated earlier, the phenomenon of nucleation, together with further growth, is one of the mechanisms by which solidification takes place, i.e. the transition from liquid to solid. The phenomenon of nucleation increases the number of crystals present in a solid aggregate, while the phenomenon accretion increases the size of these crystals. Generally, if the nucleation rate is very high compared to the speed of growth, then the solid present a structure closest to the amorphous state, while if the growth rate is very high compared to the nucleation rate, the solid will show a monocrystalline structure (more difficult to be realized). A mechanism for spontaneous grain refinement comes from the breaking of dendrites on mergers growths occurring during highly supercooled fusions. The processes of atomic rearrangement in solid-liquid interfaces, may be of the collision-limited or diffused category. This latter requires diffusive type jumps while the interface is in motion (the interface speed is given by the ratio between the liquid diffusive proprieties and the atomic diameter). In collision-limited conditions the interfacial processes are, of course, not diffusive. Great interest must be given to the heat transfer rates as these do not occur in the same manner.

As an example, the combination of slow accretions and rapid exterior loss of heat ensure that at high undercooling all the conditions of grain refinement can be sustained. In our case it can be hypothesized a final structural model based on a series of simple assumptions: a spherical domain of radius $\mathbf{r}$, the radial propagation of heat, the solid fraction function of both the semi-solid area and the subsequent one; from these assumptions it derives a front of segregation.

A particular mechanism of grain refinement is observed in highly supercooled melts of pure metals and of alloys (without partition and of collision-limited type). In such cases it is found that the size of the grain which is formed is a function of the undercooling temperature of and that, for critical $\Delta \mathrm{T}^{\star}$ values, the grain assumes small size spheroid nucleation. Arguably, this does happens for a rapid transfer of heat, which leads to a modification of the grain size for $\Delta \mathrm{T}>\Delta \mathrm{T}^{\star}$ (sub-critical cooling). The grains may contain some refined spherical elements (probably the result of a crystallization) or growths that occurred after solidification. The presence of metastable globular structures of different size indicates that the transition from the dendritic structure to that of the spheroidal segregation (and therefore to the refinement of the grain size) occurs in arrangements for rapid cooling. In addition, the disintegration of dendrites both for recasting or for shattering, contributes to the formation of redefined structures, the fragments of which would act as growth centre: this could explain the amorphous structures observed in forensic practice. These formations, owing to the lack of geometric regularity, may be related to the increase of structures to which failed the grain refinement mechanism also for the sudden evolution of the solid-liquid interface energy. This thank to the acquisition of vibration energy caused by the impacts against the cartridge case internal surface and this detail could explain why amorphous structures are seldom observed outside spent cases. The primer cup confined environment determines the genesis of primary metastable particles: said environment has such features that, for the infinitesimal time involved, can be considered as a (quasi) adiabatic container. The first (metastable) formations generated in the primer are conveyed to the vent hole and escaping in the internal of the shell may continue to aggregate and to collide on its inner surface. Subsequently, in isothermal and isobaric idealization of the phase immediately subsequent to the projectile exit from the barrel, it can be observed - as a function of its temperature of solidification- the new formations local 
undercooling and their successive nucleation, the local undercooling of the structures, their succeeding nucleation and growth in grain refinement mode (due to the local conditions the nucleation mode can be homogeneous or heterogeneous). The primitive nucleation happens because of undercooling of the elements of more high solidification temperature: the growth that derives appears diversified and, due to the mechanisms of grain refinement spheroid aggregates are more represented. The flame darts breaking through the gunpowder will induce its combustion and a ensuing piston effect on the projectile. This environment (highly chaotic) lends itself, due to the presence of the partially combusted particles (propellant), to heterogeneous nucleation [56]. This representation takes into account several mechanisms, complementary between them and consequence of very local conditions: the inner walls of the cartridge case, considering the comparative dimensions, may be considered as flat [57]. The metastable structures that are propelled out of the vent with this flat surfaces and collide for the viscosity induced by the impact, the presence of some roughness on the surface and of some embryos (developed at the impact time, of the involution of some nucleus with $\mathbf{r}<\mathbf{r}^{*}$, or of some dendritic fragments) facilitated the development of solid multiaxial aggregates as the necessary and sufficient conditions for the mechanism of grain refinement are not present (absence of spheroidal morphology).

Conversely, the metastable elements that project from the vent like a conical spray and that do not collide with the inner cartridge case surface, they assume a more regular spherical shape, as the process of heterogeneous nucleation and growth is stimulated by the presence of extraneous particles (in our case unburned or partially burned minute parts of propellant, inert grit added to the priming mixture, etc.). In addition, due to the mechanism of grain refinement (not perturbed by the collisions of the new formations against solid and irregular surfaces), the grain boundary being formed prevents the movement of dislocations along the internal symmetries typical of the crystals (the lattice structure of the grains - which differ in orientation and mutually opposes the crystalline development - because more energy is required for a dislocation to ensure the movement in the adjacent grain needs must enforce a change of direction: for these reasons, the grain boundary is much more messy than its interior, which prevents, in fact, the said dislocations). The formation of spheroids is favoured by the transfer of heat along the ideal radius, in the verse that departs from the inside of the grain through the surface that assumes an aggregation role due to the boundary structure partially not yet ordered. In the case of highly specific multicomponent particles [58], the growth is influenced by the crystalline structure of the metastable phase. This because less compact structures offer ample hollow spaces apt to accommodate additional structures: with the result that the grains fronts of growth collide with each other generating the edges of the grains. The high binding energy, the non localizability, the lack of the typical metallic bond directional properties promote, of course, the mechanisms of formation of the grain and its equiaxity.

Finally, the concomitant effects of pressure, temperature, tensity and impactivity induce additional mechanisms of recast, reaggregation and growth, mutual heat exchange with atmospheric air penetrated after the exit of the projectile; the rapid achievement of equilibrium conditions with the external (spent case in the chamber, bullet out of the barrel) freezes the structures geometry.

For the newly formed nuclei the spatial migration that refines the grain is accompanied by:

$\rightarrow$ A decrease total Gibbs energy;

$\checkmark$ A reduction of volume.

By linking the two phenomena we obtain

$$
\frac{\delta G}{\delta V}>\mathbf{0}
$$

This relationship implies that, in dimensional terms, we are faced with a force for unit of surface.

As from CNT we know that

$$
\Delta G=\frac{4}{3} \pi r^{3} \Delta G_{v}+4 \pi r^{2} \sigma
$$

we can rewrite the previous relation in terms that represent the stages of the grain refinement

$$
G=\int_{\Omega_{i}} g_{i} d V_{i}+\int_{\Psi_{i}} \sigma_{i} d S_{i}
$$

where $\Omega$ is the volume of the grain and the $\Psi$ the interface surface understood as grain boundaries.

As the total Gibbs energy $\mathbf{G}$ also decreases with the decreasing of the grain surface, then, for completed structures (spheroidal, in this case) it will be valid the following

$$
\frac{\delta G}{\delta V}=\frac{\sigma}{r}
$$


Finally, it is worth recalling that in industrial metallurgy is common practice to control the melting processes inoculating selected nucleating agents for the purpose of ensuring the proper refined, homogeneous, equiaxed and non dendritic grain structures.

A method [59] for the evaluation of grain refinement is based on the graphical representation of the relationship between grain size and a factor $\mathrm{Q}$ defined as growth restriction: this latter contains information on the efficacy of grain refining of alloys. Furthermore, it is been found [60] that the grain size can be expressed as

$$
d=\frac{1}{\sqrt[3]{\rho f}}+\frac{\Delta T_{n} b_{1}}{Q}
$$

where $\boldsymbol{\rho}$ is the density of the nucleating particles, $\mathbf{f}$ is the fraction of these activated particles, $\mathbf{b}_{1}$ a connecting factor, $\Delta \mathbf{T}_{\mathbf{n}}$ the undercooling required for nucleation. However, the above expression is just a line of type $\mathbf{d}=\mathbf{m}+(\mathbf{n} / \mathbf{Q})$ that is possible to bring on a plane $[\mathbf{d}]$ versus $[\mathbf{1} / \mathbf{Q}]$ is the variation of the angular coefficient, having fixed the intercept, and once given the slope, by varying the intercept. It shows that, in the first case, with equality of nucleating agents the grain size depends on the undercooling while, in the second case, with equal undercooling, the grain size is a function of the nucleating agents.

In these terms submicron particles, present in the primer composition No. 41 after the initiation could play, apart the previously described chemical role, also that of nucleating agent and therefore contribute to the grain refinement. In any case, for those who want to try their hand at experimental practices regarding this point, we suggest two interesting works $[61,62]$.

By the other point of view we can to interpret the phenomenon within the Time Dependent Ginzburg Landau model. The substances immersed in the rapid quenching condition do not admit homogeneous states of thermodynamic equilibrium in which coexists in contact macroscopic portions with different properties. In our case we assume that the two phases (solid-liquid) can coexist in equilibrium for a whole set of values of pressure $(\mathrm{P})$ and temperature $(\mathrm{T})$ forming a line (coexistence curve) in the plane PT.

The rapid evolution (secant to the coexistence curve), involves a transition phase. First the material is in phase A; reaching the coexistence curve there is a separation of the phases where the phase B appears and, beyond the line, phase A disappears. This implies that certain physical properties of the different substances present some discontinuity as a function of the parameters (P,T).

The rapid evolution of the physical constituents of the firing train induces phase transitions characterized by a drift of the PT relationship.

The systems class of our interest is that constituted by an evolving dynamic that we hypothesize tutored by a potential F. Since in most cases the nonlinear equations can't be exactly solved, a variational principle gives valuable information on the evolution in the time: for these systems, in fact, the dynamic power consists of an evolution towards the minimums of $\mathbf{F}$.

The description of the dynamics of a phase transition by means of a phase field is introduced for modelling the processes of solidification of pure liquids which give rise to dendritic conformation interfaces $[63,64,65]$.

The problem we face, from a thermodynamic point of view, is to assess whether the phenomenology of the GSR formation final stage is an isothermal process in the strict sense or not.

In fact, a process strictly isothermal possesses well definable characteristics that do not fit in the full sense with the phenomenology that describes the course of $\mathbf{P}(\mathbf{t})$ and $\mathbf{T}(\mathbf{t})$ during the GSR formation.

Alternatively, however, the time interval during which a cartridge is exploded (and therefore the corpuscular forms are generate) appears so small that it induces to model the phase transitions as collected in an infinitesimal time in a way that the final transient (cartridge case spent off and the bullet out of the barrel) enjoys a isothermal and isobaric regime that "freezes" what was produced during the last transition phase.

To describe the solidification (in undercooled regime) of a single-component system we will refer to an evolutionary nonconservative time dependent model (Time Dependent Ginzburg Landau), initially defining a phase variable $\boldsymbol{\Phi}$ (r,t) continuous throughout the $\boldsymbol{\Omega}$ region occupied by the system, where r represents the spatial coordinates inside of $\boldsymbol{\Omega}$, and $\mathbf{t}$ obviously the time. The variable $\Phi(\mathbf{r}, \mathbf{t})$ indicates whether the material is solid or liquid and its continuity in $\Omega$ implies that the interface solid-liquid is widespread.

By entering this latter relationship into one that provides a decrease of the total free energy, we will have that at equilibrium $\frac{\partial \phi}{\partial t}=\mathbf{0}$ it will be satisfied by the values $0 \leq \boldsymbol{\Phi}(\mathbf{r}, \mathbf{t}) \leq \mathbf{1}$ including the intermediate steps of metastable undercooled liquid or overheated solid. The thickness of the interface $\delta$ represents a compromise: the interface tends to spread to reduce the energy associated with the gradient of $\Phi(\mathrm{r}, \mathrm{t})$.

As the most representative GSR have a spherical morphology, we seek the conditions, in the Time Dependent Ginzburg Landau model, which represent a such geometry, that is to say that in a partition of $\Omega$ is generated a volume of $\theta=\frac{4}{3} \pi r^{3}$ so that a part of the undercooled liquid mass solidifies in a perfectly spherical volume. 
The curvature radius of the grain is built from a series of forces that induce the closed and curvilinear geometry during initiation of solidification, just after the nucleation: this effect can be expressed, in the processing liquid $\rightarrow$ solid, as geometric condition resulting from a pressure difference between the solid phase and the liquid phases between them divided by interface constituted by the relative thickness $\delta$.

This condition will it be represented by

$$
\mathbf{P}_{\text {solid }}-\mathbf{P}_{\text {liquid }}=\boldsymbol{\sigma} / \mathbf{r}
$$

where $\sigma$ is the free energy per unit of surface of the spherical embryo.

Furthermore, $\mathbf{r}$ satisfies the relation for which the spherical volume $\theta=\frac{4}{3} \pi r^{3}$ is given by the summation of the spatial accretions, given an assigned direction, of the areas of the circles ideals in which it is conceivable dissect the spherical volume.

Finally we represent the heat transfer formal description versus grain refinement and solidification of many types of globular structures. To escape from the improper, and unfortunately recurring, representations of the phenomena of the formation of the GSR particles, and to highlight the enormous difficulties of formalization, we represent below in a very concise manner, the basic points of its phenomenological interpretation.

Since the object of our study is fully part of, as we have stated in the first pages of this work (THE PRIMER INITIATION), the phenomena better known as Rapid Solidification Processing (RSP) with typical rate cooling of the order of $10^{3}{ }^{\circ} \mathrm{K} / \mathrm{s}$, to complete the information we recall briefly the essential points of nucleation and of equiaxed accretion in under-cooled conditions: then we'll show the basics of modelling the two types of globular structures heat dissipation, the first consisting of a sphere of liquid metal, the second consisting of a sphere of liquid metal which encompasses semi-solid material.

As extensively represented, the subcooling has a significant impact on the mechanisms of the microstructures grain refinement evolving, especially if this phenomenon takes place, in particular thermal conditions, before the solidification. The microstructures evolution (understood as successive transitions), can manifest themselves in terms of dendrites fragmentations interpretable as spread of the atomic aggregates in interdendritic liquid. After the nucleation some structures solidify (crystallization) with the subsequent, rapid, propagation (in non-equilibrium conditions) in the sub-cooled molten environment. Consequently to the rapid release of heat during this period (which clearly reveals the crystallization), the undercooled fusion heats tends to the liquid phase temperature (recalescence).

If not disturbed, the inter-dendritic liquid (that coexists with dendritic formations) will tend to solidify under conditions close to equilibrium in a certain time $t^{\text {recal }}$. The fragmentation of the dendrites is favoured if the time interval $t^{\text {break }}$ in which it manifests itself is less than the period $t^{\text {recal }}$ in which coexist the dendritic solid phase and the dendritic liquid phase in thermal evolution tending to equilibrium. In other words if the dendrites breaking time is inferior to the time of the dendrites recalescence phase, the dendrites structures have the possibility of fragmentation and crystallize in finished equiaxed microstructures.

Since the time interval $t^{\text {recal }}$ depends on how the heat is transferred to the environment, fragmentation of dendrites is more favoured by small rate cooling ( $\left.\mathrm{t}^{\text {cooling }}>\mathrm{t}^{\text {break }}\right)$ rather than by rapid heat dispersion $\left(\mathrm{t}^{\text {cooling }}<\mathrm{t}^{\text {break }}\right)$.

For the purpose of modelling, we assimilate the globular structures being in refinement mode to the ball of liquid metal initially at a uniform temperature, subsequently subject to a sudden cooling of its surface by means of heat dispersion in radiative and convective mode.

The problem of heat conduction in the idealization of the spherical symmetry can be formulated, in radial coordinates $\mathbf{r}$, by

$$
\frac{1}{r^{2}} \frac{\partial}{\partial r}\left(r^{2} \lambda_{i} \frac{\partial T_{i}}{\partial r}\right)=\rho_{i} c_{i} \frac{\partial T_{i}}{\partial t}
$$

where the subscript $i$ represents alternately the liquid phase (liq) or solid phase (sol), $\lambda$ the thermal conductivity, $\rho$ the density, $c$ the specific heat capacity. Follow here the boundary and the initial conditions

$$
\frac{\partial T}{\partial r}=\mathbf{0}
$$

for $\mathrm{r}=0$ and $t>0$

$$
-\lambda_{i} \frac{\partial T_{i}}{\partial r}=h\left(T_{i}-T_{\infty}\right)+\varepsilon_{i} \sigma\left(T_{i}^{4}-T_{i \infty}^{4}\right)
$$


for $\mathbf{r}=\mathbf{R}, \mathbf{t}>\mathbf{0}$ and $i=$ liquid (before recalescence) or $i=$ solid (after recalescence)

$\mathrm{T}=\mathrm{T}_{0}$ for $t=0$ in the range $\mathbf{0} \leq \mathbf{r} \leq \mathbf{R}$

where R represent the radium of globular structure, $\boldsymbol{\varepsilon}$ the total hemispherical emissivity, $\boldsymbol{\sigma}$ the Stefan-Boltzmann constant, $\mathbf{L}$ the latent heat, $h$ the heat transfer coefficient, finally $\infty$ it is to be understood as the parameter reported to the achievement of the environmental conditions.

The difference in density between the solid phase and the liquid phase at the interface during the phase change induces a motility of the liquid (availability of the lowest density of the Gibbs free energy of new structure).

During the phase change, at the solid/ liquid interface, since the density of the respective phases ( $\boldsymbol{\rho}_{\text {liq }}$ e $\boldsymbol{\rho}_{\text {sol }}$ ) at the melting temperature, generally, differ in a range $<10 \%$ (for which it is plausible to assume $\boldsymbol{\rho}_{\text {liq }} \approx \boldsymbol{\rho}_{\text {sol }}$ ) we can impose such boundary conditions $\mathbf{r}=\mathbf{r}_{\text {liq, }}$, $\mathbf{T}_{\text {liq }}=\mathbf{T}_{\text {sol }}$ whence

$$
-\lambda_{l i q} \frac{\partial T_{l i q}}{\partial r}+\rho_{l i q}\left(-\frac{d r_{l i q}}{d t}\right) L=-\lambda_{s o l} \frac{\partial T_{s o l}}{\partial r}
$$

It follows that the key factors that control the RSP include the energy balance, closely related to the density of the Gibbs free energy, as well as the heat extraction rate and the kinetics of nucleation and solidification.

Conversely if it semi-solid structures they hit liquid phase structures, the mixture that may result, if the first to be fully surrounded by liquid matter, can be modelled, in enthalpic terms, as follows, whereas $\boldsymbol{H}$ is the enthalpy per unit volume, $\boldsymbol{k}$ the partition coefficient,

$$
\frac{\partial H}{\partial t}=\frac{1}{r^{2}} \frac{\partial}{\partial r}\left(r^{2} k \frac{\partial T}{\partial r}\right)
$$

The enthalpy per unit volume $\mathrm{H}$ can be expressed like

$$
H=C_{p} T+\left(1+f^{s}\right) \mathrm{L}
$$

where $\mathbf{C}_{\mathbf{p}}$ represent the volumetric heat capacity, $\mathbf{f}^{\mathrm{s}}$ the solid fraction volume, $\boldsymbol{L}$ the enthalpy of fusion per unit volume.

The mathematical difficulty of the latter representation consists in the multiplicity of situations required and competitors: in fact, regardless of the solid and the liquid phases we are in the presence of extra-dendritic liquid and inter-dendritic liquid, of solid matter commingled in semisolid matter, of the two main interfaces (interface represented by inter-dendritic liquid versus solid matter with respect to the solid matter and with respect to the inter-dendritic liquid), of the borderline between extra-dendritic liquid and inter-dendritic liquid, of growth fronts between the solid matter and the extra-dendritic liquid. All these situations are, obviously, evolutionary and they needs of very particular initial conditions and boundary conditions.

In support of these modelling it is worth to mention the distribution of topographic profiles of each atomic species: in fact, the heat transfer modes have a significant influence on the localization of chemical species because of the atomic properties of the same. For example, metals having a low atomic number (i.e. atomic radius and the atomic weight lower compared to heavy metals) would be facilitated, because of convective motions, even in crossing through more dense, porous and lower temperature structures: this process promotes the migration of light metals towards the outer surface.

The presence of less dense and more fluid material in motion between the structures (in formation) at higher density would contribute to the creation of an adsorbent dynamic mode in which the denser structures may be recognized as capillaries canalicula.

Since the reactions that tend to reduce the surface free energy are, in the formation structure, thermodynamically favoured, the migration process (motion) of chemical species with low atomic number would further decrease the free energy surface. Not by chance in the idealization of the isotherm of Gibbs adsorption, the concentration of excess surface $\Gamma_{i}$ (which measures the impoverishment in the bulk of one component with respect to the enrichment of the same component on the surface) for single chemical species is defined, in terms of surface tension $\boldsymbol{\sigma}$, like

$$
\Gamma_{i}=-\frac{C_{i}}{R T} \frac{d \sigma}{d C_{i}}
$$

where $\boldsymbol{R}$ is the gas constant and $\mathbf{C}_{i}$ the concentration of a single chemical species within the particle.

This representation, in this paper timidly mentioned, provides the idea of the complexity of the formal description of GSR particles 
formation, a description that would require very serious scientific and targeted insights, consisting of theoretical and experimental studies.

To this scope, these Authors have completed the design of a "cold box" - $\mathrm{LN}_{2}$ cooled - in which, through suitable devices can be imposed, with sufficient precision, the desired subcooling: in this way who reads can try to verify the hypothesis proposed in this paper.

\section{Implications to the detection of GSR}

For the proper identification of GSR by SEM/EDX, together with a correct and fitting elemental analysis, it is also essential to take into account the particles morphology and the nature of their surfaces. GSR corpuscles are formed during a rapid solidification process and, having assumed at the liquid state a spherical shape, they maintain this characteristic as solidification occurs. Their surfaces, probably due to a phenomenon of grain refinement, should appear completely amorphous at the normal working magnification with no trace of crystalline formations. This principle has been expressed already in 1977 by G.M. Wolten and colleagues in their fundamental work [2], and has been confirmed over the years by all the experienced scientists of the field including Torre [6] and others [66].

In forensic practice it's not uncommon to find particles that possess certain characteristics: in fact sometimes some particles appear empty in several substantial part of the internal volume or display evident surface cavities. A likely scenario on the formation of these type particles is proposed here, in three points, between them interdependent, inspired by what they have just argued.

In the first case we assume that at the moment of partial solidification of globular structure (for which previous reports are met by localization in evolution topographies), if the latter are projected into the air, then partially solidified in the outer surface, pores and interstices that engulf and trap gaseous matter, vanishing immediately afterward. can be produced. A second possible explanation involves modelling such particles starting from a boundary layer that defines a spherical volume and at the decrease of the maximum radius, we identify and we define the different layers of matter (spherical surfaces) up to a liquid core. Considering the sequence of layers, and then the i-th representation of the free energy density relative to each of them, it appears likely that a core of material still in the liquid state (the temperature of the latter is non-uniform in the whole volume occupied and greater than the melting point and therefore the greater of the layers that surround it) pushes, from the inside, towards the outer layers in cooling but not yet solidified: such effects would result in an increase of the internal pressure which, successively, would induce many viscoplastic movements (of convective nature) in the layers at direct contact with the liquid mass and consequently with the intermediate ones just above. Recasting phenomena and concomitant depressive effects (induced by the masses in convective motion) could cause the appearance of trapped gas phase, gases successively vanishing. Finally a third, plausible explanation may be imagined if we consider certain zonules of the surface with an inferior thickness which represent, with respect to the remaining surface, a critical tension, and then a specific point through which the matter still liquid and under pressure extrudes outwards.

This type of particles with porosity and cracks could, most likely, be generated in areas relatively distant from the primer pocket and the flash hole.

At the end, in conflicting evidence of the literature cited from many years, for reasons we do not understand some Authors $[67,68,69]$ still describe the formation of GSR as a phenomenon that occurs with the solidification of the gases when they leave the inside of the weapon. By the way a notorious expert, operating in our country, often asserted in court that the typical GSR spherical morphology was due to the circular motion given to the particles by the barrel rifling.

\section{Conclusion}

It was then almost always acknowledged that the spheroidal morphology of GSR - particles that are formed by solidification from the droplets in their turn formed by liquefaction (or condensation) of the gases formed in the course of the initiation explosion obviously depends on the circumstance that the sphere is the three-dimensional figure with the minimum surface / volume ratio. This work, in the context of the classical theory of nucleation, presents an estimate of the critical radius of nuclei (mono elementals) that forms when a primer cap is activated. As the theory leads us to estimates much higher diametrical values in relation to what is actually found in forensic science practice, we propose to interpret the spheroids dimensional differences in the perspective of the grain refinement mechanisms of molten and undercooled metals. Furthermore, the failure of the grain refinement and the partial dendritic development could explain the different morphology of GSR: by reason of this we may suppose that the amorphous structures could be formed at the inside of the cartridge case due to collisions against the hot internal walls on which the droplets can adhere losing their shape, solidifying slowly without be interested by the grain refinement process and assuming therefore a sizeable crystal structure. Finally, it is proposed, for the spheroidal formations produced in undercooled liquid, a physicalgeometrical characterization in a not conservative time-dependent evolutionary model time dependent of the Ginzburg-Landau. It follows that it is possible, then, to connect the nucleation to the growing and refining of a solid single component structure (the multicomponent generalization obviously implies a formal and computational complication) of spheroidal type (in the phase transition liquid $\rightarrow$ solid under-cooled matter) to describe the transient final isothermal and isobaric after the projectile exit from the barrel. It is understood that the hypothesis proposed here attempts to meet the human feeling of Galilean "curiositas" on the mechanisms that govern the formation of gunshot residues: conversely, in forensic science, the excellent and so far unchallenged 
Basu's experimental work suggests that at the usual magnifications GSR appear, in most cases, as amorphous surfaced spheroidal particles. As far as this important work is concerned we have rectified the pressure and temperature values there indicated and, so far, accepted uncritically and without any hesitation. The late Dr. Basu, with great truthfulness, stated that : "The curve is not an experimental one. It has been drawn, as much as possible following curves and information on from texts [11,12] pertaining in ignition of primers and nitrocellulose" [1].

Indeed [12] 70 reference does not give information on ignition of primers and nitrocellulose while reference [11] 71 does not treat either primers or nitrocellulose. In this last work we find some pressure/time curves relating the detonation of high explosives, scantly useful for plotting deflagrations pressures or temperatures.

Further confirmations of the importance of undercooling compared to pressure jumps reside in the mentioned work [71] in fact the residual particles of the lighters compounds for morphology and dimensions may, indisputably, be associated with the GSR particles. The process of their formation due to tribological effects appear more tied to thermal jumps in the time unit.

The formation of spherical particles in common cigarette-lighters with flints usually containing iron (b.p. $2750^{\circ} \mathrm{C}$ ), cerium (b.p. 678 ${ }^{\circ} \mathrm{C}$ ), neodymium (b.p. $3068^{\circ} \mathrm{C}$ ), praseodymium (b.p. $3512^{\circ} \mathrm{C}$ ) and magnesium demonstrates that the main factor of the process is the temperature as the pressures locally developed are comparatively moderate.

Recent works [72,73] have explored the formation of particles in two other different situations, i.e. welding fume particles originating from both the core and the covering of electrodes used in popular manners of welding steel and aluminium alloy constructions and post-blast explosive particles related to both inorganic and organic reactive materials.

In the Zuzanna Brožek-Mucha work it is stated that "In samples originating from steel welding electrodes (nos. 1-9 in Table 1) the predominant group of detected particles consisted of spherules containing iron and partially crystallised iron oxide on their surface" and that: "The performed chemical and morphological examinations of particles originating from welding processes by means of SEM-EDS technique demonstrated that this human activity can be a source of a small number of spherical particles consisting of aluminium, titanium, or a set of light elements such as aluminium, silicon, potassium and calcium that are undistinguishable from gunshot residue of some kinds of modern types of ammunition".

For us it is significant that particles produced in high temperature and at normal environmental pressure situation present predominantly a spherical morphology.

In the second work it was found that Spheroidal particles $(10 \mu \mathrm{m}$ to $210 \mu \mathrm{m})$ with micro surface features recovered following inorganic charge detonations were dissimilar to the irregularly shaped particles (5 $\mathrm{m}$ to $100 \mu \mathrm{m})$ recovered following organic charge firings.

It is so unmistakable that high temperature has the predominant role also in the formation of GSR particles when a firearm is fired.

Finally, in obedience to an unwritten code of ethics, we must inform that, just before presenting this work, we had the chance to read two very interesting articles $[74,75]$ that deal with topics related to what we have hereinabove illustrate.

\section{Acknowledgements}

The authors would like to thank Peter Ross (Victoria Police Forensic Services Centre, Macleod, Victoria, Australia) and Bryan Burnett (Meixa Tech, California, USA) for their scientific revision of manuscript.

\section{References}

1. Basu S (1982) Formation of Gunshot Residues. J Forensic Sciences 27: 72-91.

2. Wolten GM, Nesbitt RS, Calloway AR, Loper GL, Jones PF (1977) Final Report on Particle Analysis for Gunshot Residue Detection. The Aerospace Corporation, El Segundo (CA).

3. Morin M (2013) Primer mixes composition and behaviour.

4. Chang LM, Williams AW (2007) Characterization of Particle Output from a Percussion Primer, ARLTR-4188.

5. Morin M, Hreglich S, Verità M (1982) Alcune considerazioni sullimpiego pratico del microscopio elettronico a scansione con spettrometro a raggi X per la ricerca e per l'identificazione dei residui di sparo Convegno di Balistica Forense Roma.

6. Torre C, Mattutino G, Vasino V, Robino C (2002) Brake Linings: A Source of Non-GSR Particles Containing Lead, Barium, and Antimony. J Forensic Sci 47: 494-504.

7. Bowden FP, Yoffe YD (1952) Initiation and growth of explosion in liquids and solids, Cambridge.

8. Matyáš R, Šelešovský J, Musil T (2012) Sensitivity to friction for Primary Explosives. J Hazard Mater 213-4: 236-41.

9. Wu YQ, Huang FL (2011) A microscopic model for predicting hot-spot ignition of granular energetic crystals in response to drop-weight impacts, Mechanics of Materials 43: 835-852.

10. Langer G, Eisenreich N (1999) Hot spots in energetic materials. Propellants, explosives, pyrotechnics 24: 113-118.

11. Jette FX (2012) On relationship between shock and thermal initiating conditions for various reactive powder mixtures. Propellants, explosives, pyrotechnics 37: $345-58$.

12. Afanas'ev G-T, Bobolev VK (1968) Initiation of solid explosives by impact, Academy of Sciences of the USSR - Institute of Chemical Physics, Moscow. 
13. Kholevo NA (1946) New data on the sensitivity of condensed explosives to mechanical shock. Kazan Chemical Engineering Institute 10: 91-106.

14. Wenograd J (1961) The behaviour of explosives at very high temperatures, U.S. Naval Ordnance Laboratory, NAVWEPS Report 7328.

15. Gonor A, Hooton I, Narayan S (2002) Steady-state model of heterogeneous detonation with reactive metallic particles Proceedings of the Twelfth International Detonation Symposium August 11-16.

16. Howard WM, Fried LE, Souers PC (2000) Modelling of non-ideal aluminized explosives. AIP Conference Proceedings 505: 389.

17. Zhang Q, Chang Y (2012) Prediction of Detonation Pressure and Velocity of Explosives with Micrometer Aluminium Powders, Central European. J Energ Mater 9: 77-86.

18. Friedman MH (1963) Prediction of detonation characteristics of condensed explosives, Propellant Research Laboratory Meeting of the American Chemical Society, New York.

19. Dikinson M, Field JE (1993) Initiation and propagation in primary explosives. Mathematical and Physical Sciences 441: 359-275.

20. AA VV (1970) Percussion primers, design requirements, McDonnell Douglas Corporation, Report MDC A0514.

21. DTR (1977) Experimental study of transient combustion processes in granular propellant beds, M.S. Thesis, The Pennsylvania State University.

22. Kuo KK, Moore BB, Ghent DY (1979) Characterization of Mass Flow Rates for Various Percussion Primers. Seventh ICOGER Conference 20-24.

23. Wu CJ, Fried LE (2000) First-Principles Study of High Explosive Decomposition Energetics. Proceedings of the 11th International Symposium Detonation 6: 490.

24. Rice BM, Hare JJ (2002) A Quantum Mechanical Investigation of the Relation between Impact Sensitivity and the Charge Distribution in Energetic Molecules. J Phys Chem A 106: 1770-783.

25. Bauer A, Cook MA, Keyes RT (1961) Detonation-generated plasmas. Mathematical and Physical Sciences 259: 508-17.

26. Chang LM, Williams AW (2007) Characterization of Particle Output From a Percussion Primer, ARLTR-4188.

27. Robert M, Jiří P (2013) Primary Explosives. Springer.

28. SE Stephenson (1986) A technique for investigation of ignition phenomena in small arms ammunition Department of Defence - Defence Science and Technology Organization Weapons Systems Research Laboratory Defence Research Centre Salisbury South Australia.

29. Buckle ER (1986) Particle condensation in the vapour emitted by a heated source. J colloid interfaces Sci 112.

30. Glicksman ME (1965) Dynamic effect arising from high-speed solidification. Acta Metallurgica 13: 1231-46.

31. Courtney WG (1963) Homogeneous nucleation from simple and complex system AIAA Heterogeneous Combustion Conference, Palm Beach, Forida, December 11-3.

32. Dunning W (1963) Experimental methods for the study of nucleation and condensation AIAA Heterogeneous Combustion Conference, Palm Beach, Forida, December 11-3.

33. Kathmann SM (2006) Understanding the chemical physics of nucleation. Theor Chem Acc 116: 169-82.

34. Quian M (2007) Heterogeneous nucleation on potent spherical substrates during solidification. Acta Materialia 55: 943-53.

35. Good RJ (1976) Surface free energy of solids and liquids: thermodynamics, molecular forces and structure. J Collo Interf Sci 59: 398-419.

36. Shimizu I, Y Takey (2005) Thermodynamics of interfacial energy in binary metallic systems: influence of adsorption on dihedral angles. Acta Materialia 53: $811-21$.

37. Mondal K, Kumar A, Gupta S, Murty BS (2009) Temperature and structure dependency of solid-liquid interfacial energy. Acta Materialia 57: 3422-30.

38. Jones $\mathrm{H}$ (2002) The solid-liquid interfacial energy of metals: calculations versus measurements. Materials Letters 53: 364-6.

39. Liu J, Davidchack RL, Doing HB (2013) Molecular dynamics calculation of solid-liquid interfacial free energy and its anisotropy during iron solidification. Computa Mater Sci 74: 92-100.

40. Jiang Q, Lu HM (2008) Size dependent interface energy and its application. Surf Sci Rep 63: 427-64.

41. Quian M (2007) Heterogeneous nucleation on potent spherical substrates during solidification. Acta Materialia 55: 943-53.

42. Dubey KS, Ramachandrarao P (1984) On the free energy change accompanying crystallization of under cooled melts. Acta Metall 32: 91-96.

43. Willnecker R, Herlach DM, Feuerbacher B (1986) Containerless undercooling of bulk Fe-Ni melts. Appl Phys Lett 49: 1339-41.

44. Y Kuzuokaa, S Isomaeb, Y Yamaguchia (2007) Crystal morphology of spherical silicon particles produced by jet-splitting method. J Crystal Growth 304: 487491.

45. Herlach D (2011) Crystal nucleation and dendrite growth of metastable phases in undercooled melts. J Alloys Compounds 509: S13-S17.

46. WiIInecker R, Herlach DM, Feuerbacher B (1988) Nucleation in bulk undercooled nickel-base Alloys. Mater Sci Eng 98: 85-88.

47. Perepezko JH (1984) Nucleation in Undercooled Liquids. Mater Sci Engineering 65: 125-35.

48. XJ Han, Wang N, Wei B (2002) Rapid eutectic growth under container less condition Applied Physics Letters 81: 778.

49. Herlach DM, Galenko PK (2007) Rapid solidification: in situ diagnostics and theoretical modelling. Mater Sci Engin 34-41.

50. MC Flemings, Shiohara Y (1984) Solidification of Undercooled Metals. Materials Science and Engineering 65: 157-70.

51. FP Dai, WJ Xie, Wei B (2009) Spherical ternary eutectic cells formed during free fall. Philosophical Magazine Letters 89: 170-7.

52. Michael OT (1986) Rapid solidification studies of a model alloy system. Appl Phys Lett 49.

53. Wiilnecker R, Herlach DM, Feuerbacher B (1990) Grain refinement induced by a critical crystal growth velocity in undercooled melts. Appl Phys Lett 56.

54. Dubey KS, Ramachandrarao P, Lele S (1996) Thermodynamics and viscous behaviour of undercooled liquids. Thermodinamica Acta 280-281: 25-62.

55. Maxwell I, Hellawell A (1974) A simple model for grain refinement during solidification. Acta Metallurgica 23: 229-37.

56. Mullis A (1997) A model for spontaneous grain refinement in alloy systems at low undercooling. Mater sci engine 226: 804-8.

57. Mullis A, Cochrane R (2001) A phase field model for spontaneous grain refinement in deeply undercooled metallic melts. Acta Materalia 49: 2205-14.

58. Quian M (2007) Heterogeneous nucleation on potent spherical substrates during solidification. Acta Materialia 55: 943-53.

59. Easton MA, DH StJohn (2001) A model of grain refinement incorporating alloy constitution and potency of heterogeneous nucleant particles. Acta Materalia 
49: $1867-78$.

60. Schmid-Fetzer R, Kozlov A (2011) Thermodynamic aspects of grain growth restriction in multicomponent alloy solidification. Acta Materialia 59: 6133-44. 61. St John DH, Cao P, Qian M, Easton MA (2007) A New analytical approach to reveal the mechanisms of grain refinement. Advanced Engineering Materials 9: 739-46.

62. Easton MA, St John DH (2005) An analysis of the relationship between grain size, solute content, and the potency and number density of nucleant particles. Metall Mater Trans 36: 1911-20.

63. Perepezko JH (1990) Solidification of Highly Undercooled Liquid Droplets University of Wisconsin-Madison Dept. of Materials Science and Engineering ADA218 776 .

64. Visintin A (1997) Two-scale model of phase transitions. Physica D: Nonlinear Phenomena 106: 66-80.

65. Boettinger WJ, Warren JA, Beckermann C, Karma A (2002) Phase-field simulation of solidification, Annual Review of Materials Research 32: 163-94.

66. Meidani H, Desbiolles JL, Jacot A, Rappaz M (2012) Three-dimensional phase-field simulation of micropore formation during solidification: morphological analysis and pinching effect. Acta Materialia 60: 2518-27.

67. Illker Kara, Yasin Sarikavak, Sefer Bora Lisesivdin Mehmet Kasap (2016) Evaluation of morphological and chemical differences of gunshot residues in different ammunitions using SEM/EDS technique. Environmental Forensics 17: 68-79.

68. Gallusser A, Bonfanti M, Schutz F (2002) Expertise des armes à feu et des éléments de munitions dans l'investigation criminelle 129.

69. Schwoeble AJ, Exline DL (2000) Current Methods in Forensic Gunshot Residue Analysis. Forensic Gunshot Residue Analysis 192.

70. Wolten GM, Nesbitt RS (1980) On the Mechanism of Gunshot Residue Particle Formation. Journal of Forensic Sciences 25: 533-45.

71. Cook MA (1963) The Science of High Explosives. American Chemical Society Monograph Series, Reinhold Publishing Corp., New York, 3rd printing 123-205.

72. Hsien-Hui Meng, Chun-Hung Lin (2008) Particle analysis of lighter flint residues using scanning electron microscopy/energy dispersive X-ray spectrometry. Forensic Science Journal 7: 37-44.

73. Abdul-Karim, Nadia (2016) Morphological Variations of Explosive Residue Particles and Implications for Understanding Detonation Mechanisms. Anal Chem 88: 3899-908.

74. Yang Jan, Gunn A, Palmbach T, Dongguang Wei, Sinha S (2006) Nano-Forensics---- Nanoparticles in Gun Shot Residues. Emerging Technologies - Nanoelectronics IEEE Conference 269-72.

75. Lis GA Melo, Andrea M, Andre' L Pinto (2014) Nano characterization of gunshot residues from Brazilian ammunition. Forensic Science International 240: 69-79. 